Review Article

\title{
Efficacy and Safety of Xue-Fu-Zhu-Yu Decoction for Patients with Coronary Heart Disease: A Systematic Review and Meta-Analysis
}

\author{
Shuo Zhang $\mathbb{D},{ }^{1,2}$ Zhen-Lin Chen $\mathbb{D},{ }^{3}$ Yu-Ping Tang $\mathbb{( D},{ }^{4}$ Jin-Long Duan $\mathbb{D}^{2}$, \\ and Kui-Wu Yao $\mathbb{D i D}^{2}$ \\ ${ }^{1}$ School of Clinical Medicine (Guang'anmen Hospital), Beijing University of Chinese Medicine, Beijing, China \\ ${ }^{2}$ Guang'anmen Hospital, China Academy of Chinese Medical Sciences, Beijing, China \\ ${ }^{3}$ International Programs Office, Shaanxi University of Chinese Medicine, Xi'an, Shaanxi, China \\ ${ }^{4}$ Key Laboratory of Shaanxi Administration of Traditional Chinese Medicine for TCM Compatibility, \\ Shaanxi University of Chinese Medicine, Xi'an, Shaanxi, China
}

Correspondence should be addressed to Yu-Ping Tang; yupingtang@sntcm.edu.cn

Received 5 March 2021; Revised 29 May 2021; Accepted 15 August 2021; Published 29 September 2021

Academic Editor: Jun-Yong Choi

Copyright $\odot 2021$ Shuo Zhang et al. This is an open access article distributed under the Creative Commons Attribution License, which permits unrestricted use, distribution, and reproduction in any medium, provided the original work is properly cited.

Objective. To systematically evaluate the efficacy and safety of XFZYD for coronary heart disease (CHD). Methods. A comprehensive literature search of randomized controlled trials using XFZYD for CHD was conducted in 10 electronic databases from their establishment to December 20, 2020. The researchers screened the relevant trials in NoteExpress, extracted the data in duplicate independently, assessed the risk of bias in the trials using the Cochrane collaboration tool, and then used Rev Man 5.3 for data analysis. Results. 30 trials with 3126 participants were included for meta-analysis. The results showed that the clinical effects of XFZYD and its combination with chemical drugs (CD) were 1.13 (RR; 1.13; 95\% CI, 1.03 to 1.24 ) and 1.26 (RR; $1.26 ; 95 \%$ CI, 1.20 to 1.32) times those of $\mathrm{CD}$, respectively. And, it could also improve electrocardiogram effect, which was 1.63 (RR; $1.63 ; 95 \% \mathrm{CI}, 1.04$ to 2.53) times that of CD. XFZYD could not only decrease duration of angina pectoris and improve vascular endothelial function but also obviously reduce the TCM syndrome score. When used in combination with CD, it could also lower AF, correct the dyslipidemia, and reduce the blood viscosity. Conclusion. These results demonstrated that XFZYD had great advantages in treating CHD with no obvious adverse reactions. Therefore, it is believed that XFZYD is more suitable for CHD patients with clinical indicators of dyslipidemia, high blood viscosity, or vascular endothelial dysfunction. This study is the first systematic review and meta-analysis with some unique ways, including its comprehensiveness, large-scale search, the novelty of findings, and transparent approach.

\section{Introduction}

Coronary heart disease (CHD) is a heart disease caused by atherosclerotic lesions in the coronary arteries, which causes stenosis or obstruction of the vascular lumen, resulting in myocardial ischemia, hypoxia, or necrosis [1], and it is the main cause of death worldwide. Due to its high morbidity and mortality, CHD has gradually become an important public health problem worldwide, and it has attracted extensive attention at home and abroad. Standardized treatments include drug and revascularization therapy. Although some drugs can delay the development of CHD to a certain extent, long-term medication will cause adverse reactions; for example, depression is a side effect of medication, which may aggravate the development of CHD [2].

Traditional Chinese medicine (TCM) has long-term practice in the treatment of $\mathrm{CHD}$, which belongs to the category of "chest pain" and "heartache" in TCM. Its onset is mostly caused by cold pathogen invasion, eating disorder, emotional disorder, weary internal injuries, and aging physical weakness, which leads to abnormal circulation of blood and Qi, and its pathogenesis is blockage of the cardiac vessels. Xue-Fu$Z h u-Y u$ decoction (XFZYD) has the effects of promoting blood circulation for removing blood stasis and regulating qi-flowing 
for relieving pain, which is a classic TCM formula for the treatment of blood stasis syndrome [3] and is widely used in a variety of cardiovascular diseases [4]. XFZYD is composed by 11 kinds of herbs, including Persicae Semen (Taoren), Carthami Flos (Honghua), Aurantii Fructus (Zhiqiao), Radix Platycodonis (Jiegeng), Medicinal cyathula root (Chuuanniuxi), Bupleuri Radix (Chaihu), Rehmanniae Radix (Shengdi), Angelicae Sinensis Radix (Danggui), Chuanxiong Rhizoma (Chuanxiong), Paeoniae Radix Rubra (Chishao), and Glycyrrhizae Radix Et Rhizoma (Gancao). Although there were many randomized controlled trials in the clinic to study the efficacy of XFZYD in the treatment of CHD, the research was fragmented and lacks systematization. Moreover, due to different sample sizes, usage and dosage, and outcome indicators, the evaluation criteria were not unified, and the research conclusions were not consistent. In addition, although there were several metaanalyses on the treatment of CHD with XFZYD currently [5-7], they only focused on one aspect of angina and did not include other symptoms or clinical classifications of CHD. Therefore, the comprehensiveness of XFZYD in the treatment of CHD cannot be obtained. In conclusion, this study aimed to comprehensively evaluate the efficacy and safety of XFZYD for CHD through systematic reviews and meta-analysis, and to summarize its potential therapeutic mechanisms, which was the first most large-scale and extensive research in this field, and the results will provide more effective and credible evidence for further guiding clinical practice and fill the knowledge gap of XFZYD for precise medication of CHD.

\section{Data and Methods}

This study was conducted and reported in accordance with the Preferred Reporting Items for Systematic Reviews and Meta-analyses (PRISMA) with Cochrane methodology [8]. This study has been registered, and the PROSPERO number is CRD42021229958.

2.1. Literature Search. From the establishment of each electronic database to December 20, 2020, randomized controlled trials that evaluated the effect of XFZYD for treating $\mathrm{CHD}$ were searched in the following 10 electronic databases: Cochrane Library (from 1966 to 2020), Medline (from 1946 to 2020), PubMed (from 1959 to 2020), Springerlink (from 1996 to 2020), Web of Science (from 1986 to 2020), ClinicalTrials.gov, Chinese Biomedical Literature Database (CBM, from 1978 to 2020), the Chinese National Knowledge Infrastructure (CNKI, from 1980 to 2020), Wanfang Database (from 1998 to 2020), Weipu Database (from 1989 to 2020), and Chinese Biomedical Literature Database (CBM, from 1978 to 2020). Forward and backward citation searching was conducted for all eligible trials. The following terms were used for searching: ("coronary heart disease" OR "xiongbi" OR “xintong”) AND ("xuefuzhuyu decoction" OR "xuefu zhuyu decoction" OR "xue fu zhu yu decoction" OR "xuefuzhuyutang" OR "xuefuzhuyu tang" OR "xuefu zhuyu tang" OR "xue fu zhu yu tang") AND ("clinical trial" OR "randomized controlled trial" OR "randomized controlled trial" OR "lin chuang yan jiu" OR "lin chuang shi yan"). The language and status of publications in our literature search were not be specified. And we manually searched bibliographies of included trials and related reviews for additional references.

\subsection{Criteria for Literature Inclusion}

2.2.1. Type of Research. This study included randomized controlled trials of XFZYD in the treatment of CHD. Trials were excluded if (a) they were not random; (b) no control group was used; (c) XFZYD was not used in the experimental group; (d) they are combined with other drugs; (e) experimental design was not rigorous, or statistical methods were inappropriate in trials; (f) they had indeterminacy of measurement index outcome criterion; (g) trials on effective analysis data cannot be obtained; (h) they are reviews, conference paper, case reports, experience sharing, etc.; (i) they are animal experiments; $(j)$ they are repeatedly published articles and plagiarized studies.

2.2.2. Study Subjects. Patients who were not restricted by age, gender, or nationality with $\mathrm{CHD}$ were eligible for inclusion in this study.

2.2.3. Intervention Measures. The intervention measures in the experimental group should be XFZYD (no herb added or subtracted) or combined with the treatment of control group. And the control group should be chemical drugs (CD).

2.2.4. Outcomes. The primary outcome was defined as angina frequency (AF) and duration of angina pectoris (DAP). The secondary outcomes were angina, which included clinical effect; electrocardiogram effect; blood lipid, which included total cholesterol (TC), triglyceride (TG), low-density lipoprotein cholesterol (LDL-C), and highdensity lipoprotein cholesterol (HDL-C); hemorheology index, which included whole blood viscosity (WBV), plasma viscosity (PV), and fibrinogen (FB); vascular endothelial function indicators, which included $\mathrm{NO}$ and endothelin (ET); TCM syndrome score, and adverse reaction.

2.3. Study Selection and Data Extraction. According to the inclusion and exclusion criteria mentioned above, two researchers who participated in calibration and training exercises before starting the screening processes independently screened the titles and abstracts of potential eligible trials, which were in duplicate, and then they retrieved independently and reviewed the full text of the possible trials in duplicate based on the inclusion and exclusion criteria and compared their results. The screening process was conducted in Note Express 3.2.0.

We conducted various forms of calibration exercises and pilots before the data extraction process began. Two researchers used standardized tables to independently extract data in duplicate from all eligible trials according to the inclusion and exclusion criteria mentioned above. In 
case of disagreement, they agreed through discussion or submitted it to a third party for evaluation. And before the screening process, the third party used a standardized screening form and performed calibration exercises.

(1) The basic information of the study (author's name, title of the study, year of publication, country/region, and publication status)

(2) Study characteristics (sample size, source of cases, age, diagnostic criteria, and inclusion and exclusion criteria)

(3) Intervention and control measures (dosage form, dose, and duration)

(4) Research methodology (random scheme generation, allocation hiding, blind method, incomplete result data, selective reporting, other biases, and loss of follow-up)

(5) Outcome measures

2.4. Assessment of Literature Quality. The methodological quality of each included study was assessed independently by two reviewers according to the Cochrane collaboration tool. It comprised the following 7 aspects: random sequence generation, allocation concealment, blind method, incomplete result data, selective reporting, and other biases. The quality assessment results of each item can be divided into three grades: "low risk," "high risk," and "unclear." The more rigorous the design and the higher the methodological quality of each RCT, the lower the risk coefficient. For example, according to the random control table, the random sequence generation is "low risk." Furthermore, the RCT that used allocation concealment and blind methods without incomplete outcome data, selective reporting, or other biases was considered as "low risk"; otherwise, it will be considered as "high risk." If none of the above 7 aspects was reported in the trial, the aspect was considered to be "unclear." When necessary, the consensus on this issue was studied with the help of a third party.

2.5. Statistical Analysis. Data analysis was performed using Rev Man 5.3 software. Both the continuous and dichotomous outcomes were derived from the included trials without any conversion. The dichotomous outcomes were described by relative risk (RR) and 95\% confidence interval (CI); in addition, mean difference (MD) and 95\% CI were used to describe the effect value of the intergroup comparison. Heterogeneity was determined according to the results of $I^{2}$ test. $I^{2}<50 \%$ indicated the low heterogeneity of interstudy, and the fixed effect model was adopted. Furthermore, the random effect model was adopted when $I^{2}>50 \%$ [9]. Random effect model was also used to generate direct and mixed treatment comparison estimates. Subgroup analysis was conducted according to whether the experimental group was combined with chemical medicine and the different treatment methods in the control group. Inverted funnel plots were used to determine publication bias when the number of included studies exceeded 10 in the metaanalysis [10].

\section{Results}

3.1. Results of Our Literature Search. Based on the above retrieval strategy, a total of 1880 potentially relevant trials were retrieved from 10 electronic databases, and 657 trials were retrieved after 1223 duplicates were deleted. After reviewing the titles and abstracts, 434 trials were excluded, because they did not comply with the inclusion criteria, and 223 trials initially met the predetermined requirements, and their full texts were read for detailed assessment. Finally, 30 trials were included for meta-analysis [11-40]. The PRISMA flow diagram of literature retrieval process is shown in Figure 1. All included trials have been published as full article.

3.2. Basic Characteristics of the Included Studies. Table 1 summarizes the basic characteristics of the eligible 30 trials and analyzed a total of 3126 patients with CHD. Sample sizes ranged from 40 to 300 . In the included trials, XFZYD combined with chemical drug (XCWC) vs. chemical drug (CD) was used in 26 trials, and XFZYD vs. CD was used in 4 trials. In primary outcomes, 9 trials reported AF and 8 reported DAP. In terms of secondary outcome indicators, 22 trials reported clinical effect, 8 reported electrocardiogram effect, 9 reported TC, 6 reported TG, 8 reported LDL-C and HDL-C, 2 reported WBV, 3 reported $\mathrm{PV}$ and $\mathrm{FB}, 3$ reported $\mathrm{NO}$ and ET, 4 reported TCM score, and 6 reported adverse effects. Duration of treatment was reported in all included trials from 14 days to 1 year.

3.3. Risk of Bias Assessment of the Literature Included in the Study. The methodological quality of 30 eligible trials is summarized in Figures 2-3, and the criteria in the Cochrane Handbook for Systematic Reviews of Interventions were used to assess the risk of bias in the study. Although randomization was announced in all of the included trials, 4 trials did not report the adequate sequence generation. 26 trials used random number table [11-29, 31-37], and 1 used block randomization [30]. 3 trials reported blind method [38-40], but only 1 trial reported allocation concealment [25]. Figure 4 provides the funnel plot of the trials for clinical effect, and Egger's test calculated $p=0.005$, which revealed that there may be some publication bias in the trials.

\subsection{Outcomes}

3.4.1. Angina (AF and DAP). 9 trials [15, 17, 19, 26, $27,29,31,37]$ reported the AF before and after intervention. Compared with CD, XCWC exhibited significant lowering effects on AF ( 9 trials; $n=1349$; MD, $-1.01 ; 95 \% \mathrm{CI},-1.31$ to -0.71 ; Figure $5(\mathrm{a}))$.

DAP was reported in 8 trials $[15,17,19,26,27,29,31$, $34]$, and the shorter the DAP, the less pain in patients. Metaanalysis evaluated a great lowering effect of XFZYD on DAP, and XFZYD could remarkably reduce DAP and relieve the 


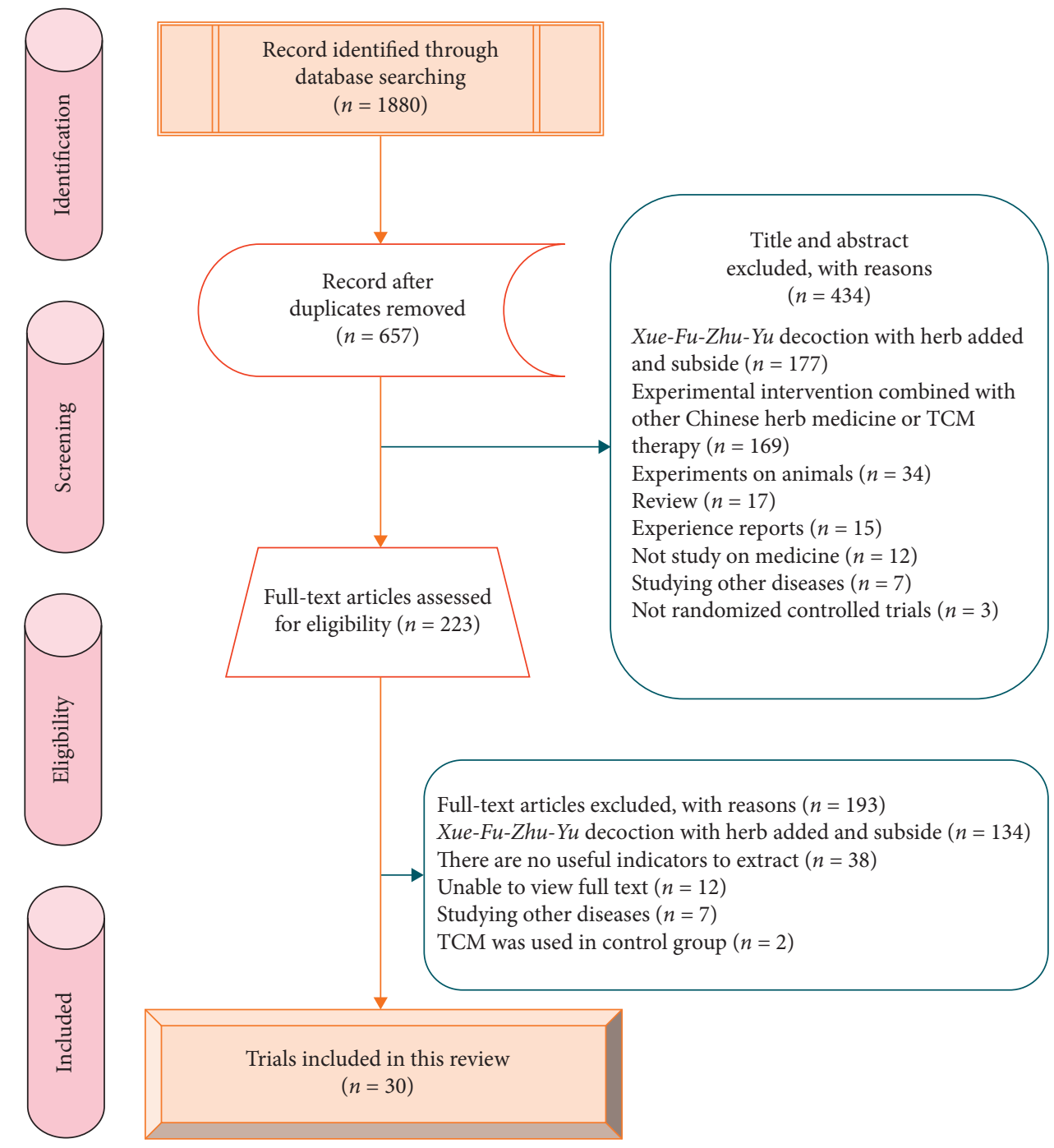

FIgURE 1: PRISMA flow diagram of literature selection.

TABLE 1: Characteristic of the 30 trials included in the meta-analysis.

\begin{tabular}{|c|c|c|c|c|c|c|c|}
\hline Author(s) & $\begin{array}{l}\text { Country and } \\
\text { setting }\end{array}$ & $\begin{array}{l}\text { Sample size } \\
\text { (experimental/ } \\
\text { control) }\end{array}$ & $\begin{array}{l}\text { Patient } \\
\text { enrollment time }\end{array}$ & Experimental & Control & Duration & Outcome measures \\
\hline An & $\begin{array}{c}\text { Liaohe Oilfield } \\
\text { General } \\
\text { Hospital, } \\
\text { Liaoning } \\
\text { Province, China }\end{array}$ & $36 / 36$ & $2017.1-2018.1$ & $\begin{array}{l}\text { XFZYD + isosorbide } \\
\text { mononitrate }\end{array}$ & $\begin{array}{l}\text { Isosorbide } \\
\text { mononitrate }\end{array}$ & 1 month & $\begin{array}{l}\text { Clinical effect; } \\
\text { TCM syndrome } \\
\text { score }\end{array}$ \\
\hline $\begin{array}{l}\text { Chen } \\
\text { et al. }\end{array}$ & $\begin{array}{c}\text { Beijing Daxing } \\
\text { District People's } \\
\text { Hospital, } \\
\text { Beijing, China } \\
\end{array}$ & $75 / 75$ & 2009.1-2011.6 & $\begin{array}{c}\text { XFZYD + conventional } \\
\text { therapy }\end{array}$ & $\begin{array}{c}\text { Conventional } \\
\text { therapy }\end{array}$ & 1 month & $\begin{array}{l}\text { Clinical effect; } \\
\text { electrocardiogram } \\
\text { effect; TC; TG; } \\
\text { LDL-C; HDL-C }\end{array}$ \\
\hline Dong & $\begin{array}{c}\text { Bei'an First } \\
\text { People's } \\
\text { Hospital, } \\
\text { Heilongjiang } \\
\text { Province, China }\end{array}$ & $40 / 40$ & $2014.5-2015.6$ & $\begin{array}{c}\text { XFZYD + conventional } \\
\text { therapy }\end{array}$ & $\begin{array}{c}\text { Conventional } \\
\text { therapy }\end{array}$ & $\begin{array}{c}3 \\
\text { months }\end{array}$ & TC; LDL-C \\
\hline
\end{tabular}


TABle 1: Continued.

\begin{tabular}{|c|c|c|c|c|c|c|c|}
\hline Author(s) & $\begin{array}{l}\text { Country and } \\
\text { setting }\end{array}$ & $\begin{array}{c}\text { Sample size } \\
\text { (experimental/ } \\
\text { control) }\end{array}$ & $\begin{array}{c}\text { Patient } \\
\text { enrollment time }\end{array}$ & Experimental & Control & Duration & Outcome measures \\
\hline Gao et al. & $\begin{array}{c}\text { Harbin First } \\
\text { Hospital, } \\
\text { Heilongjiang } \\
\text { Province, China }\end{array}$ & $38 / 38$ & 2017.3-2018.3 & $\begin{array}{l}\text { XFZYD + atorvastatin; } \\
\text { aspirin; isosorbide } \\
\text { mononitrate }\end{array}$ & $\begin{array}{l}\text { Atorvastatin; } \\
\text { aspirin; } \\
\text { isosorbide } \\
\text { mononitrate }\end{array}$ & 1 month & $\begin{array}{c}\text { Clinical effect; NO; } \\
\text { ET }\end{array}$ \\
\hline Huang & $\begin{array}{c}\text { General } \\
\text { Hospital of } \\
\text { Shenyang } \\
\text { Military Region, } \\
\text { Liaoning } \\
\text { Province, China }\end{array}$ & $20 / 20$ & $2016.1-2018.1$ & XFZYD + rosuvastatin & Rosuvastatin & 1 month & $\begin{array}{l}\text { Clinical effect; TC; } \\
\text { TG; LDL-C; HDL- } \\
\text { C }\end{array}$ \\
\hline $\begin{array}{l}\text { Kong and } \\
\text { Liu }\end{array}$ & $\begin{array}{c}\text { ShunDe } \\
\text { Hospital } \\
\text { Guangzhou } \\
\text { University of } \\
\text { Chinese } \\
\text { Medicine, } \\
\text { Guangdong } \\
\text { Province, China }\end{array}$ & $32 / 32$ & 2017.1-2018.9 & $\begin{array}{l}\text { XFZYD + warfarin; } \\
\text { atorvastatin; } \\
\text { metoprolol; aspirin; or } \\
\text { clopidogrel }\end{array}$ & $\begin{array}{l}\text { Warfarin; } \\
\text { atorvastatin; } \\
\text { metoprolol; } \\
\text { aspirin; or } \\
\text { clopidogrel }\end{array}$ & $\begin{array}{c}2 \\
\text { months }\end{array}$ & Clinical effect \\
\hline $\mathrm{Li}$ & $\begin{array}{c}\text { Shaodong } \\
\text { County } \\
\text { Hospital of } \\
\text { Traditional } \\
\text { Chinese } \\
\text { Medicine, } \\
\text { Hunan } \\
\text { Province, China }\end{array}$ & $45 / 45$ & $2008.1-2010.1$ & $\begin{array}{c}\text { XFZYD + aspirin; } \\
\text { isosorbide mononitrate; } \\
\text { simvastatin }\end{array}$ & $\begin{array}{l}\text { Aspirin; } \\
\text { isosorbide } \\
\text { mononitrate; } \\
\text { simvastatin }\end{array}$ & 1 month & $\begin{array}{l}\text { Electrocardiogram } \\
\text { effect; AF }\end{array}$ \\
\hline $\mathrm{Li}$ & $\begin{array}{l}\text { Inner Mongolia } \\
\text { Zhalantun city } \\
\text { Hospital, } \\
\text { Neimenggu } \\
\text { Province, China }\end{array}$ & $126 / 125$ & 2010.6-2012.6 & $\begin{array}{c}\text { XFZYD + conventional } \\
\text { therapy }\end{array}$ & $\begin{array}{l}\text { Conventional } \\
\text { therapy }\end{array}$ & $\begin{array}{c}3 \\
\text { months }\end{array}$ & AF; DAP \\
\hline $\mathrm{Li}$ & $\begin{array}{c}\text { Maoming } \\
\text { Hospital of } \\
\text { Traditional } \\
\text { Chinese } \\
\text { Medicine, } \\
\text { Guangdong } \\
\text { Province, China }\end{array}$ & $30 / 30$ & 2016.6-2017.6 & $\begin{array}{c}\text { XFZYD + conventional } \\
\text { therapy }\end{array}$ & $\begin{array}{c}\text { Conventional } \\
\text { therapy }\end{array}$ & 14 days & Adverse reaction \\
\hline $\mathrm{Li}$ and $\mathrm{Yu}$ & $\begin{array}{c}\text { Yueyang } \\
\text { Hospital of } \\
\text { Traditional } \\
\text { Chinese } \\
\text { Medicine, } \\
\text { Hunan } \\
\text { Province, China }\end{array}$ & $30 / 30$ & 2015.12-2016.2 & XFZYD & $\begin{array}{l}\text { Aspirin; } \\
\text { isosorbide } \\
\text { mononitrate; } \\
\text { atorvastatin }\end{array}$ & 2 weeks & $\begin{array}{l}\text { Clinical effect; } \\
\text { adverse reaction }\end{array}$ \\
\hline $\begin{array}{l}\text { Liang and } \\
\mathrm{Li}\end{array}$ & $\begin{array}{c}\text { Central People's } \\
\text { Hospital of } \\
\text { Tengzhou City, } \\
\text { Shandong } \\
\text { Province, China }\end{array}$ & $150 / 150$ & 2016.10-2018.6 & $\begin{array}{c}\text { XFZYD + aspirin; } \\
\text { isosorbide mononitrate }\end{array}$ & $\begin{array}{c}\text { Aspirin; } \\
\text { isosorbide } \\
\text { mononitrate }\end{array}$ & 1 month & $\begin{array}{l}\text { Clinical effect; AF; } \\
\text { DAP; adverse } \\
\text { reaction }\end{array}$ \\
\hline $\begin{array}{l}\text { Liao and } \\
\text { Liang }\end{array}$ & $\begin{array}{c}\text { Maoming } \\
\text { Hospital of } \\
\text { Traditional } \\
\text { Chinese } \\
\text { Medicine, } \\
\text { Guangdong } \\
\text { Province, China }\end{array}$ & $40 / 40$ & 1999.5-1999.9 & XFZYD & $\begin{array}{l}\text { Isosorbide } \\
\text { nitrate }\end{array}$ & 1 month & $\begin{array}{l}\text { Clinical effect; } \\
\text { electrocardiogram } \\
\text { effect; TC; TG; } \\
\text { LDL-C; HDL-C; } \\
\text { adverse reaction }\end{array}$ \\
\hline
\end{tabular}


TABle 1: Continued.

\begin{tabular}{|c|c|c|c|c|c|c|c|}
\hline Author(s) & $\begin{array}{l}\text { Country and } \\
\text { setting }\end{array}$ & $\begin{array}{c}\text { Sample size } \\
\text { (experimental/ } \\
\text { control) }\end{array}$ & $\begin{array}{c}\text { Patient } \\
\text { enrollment time }\end{array}$ & Experimental & Control & Duration & Outcome measures \\
\hline Liu et al. & $\begin{array}{c}\text { Chongqing } \\
\text { Jiangjin District } \\
\text { Hospital of } \\
\text { Traditional } \\
\text { Chinese } \\
\text { Medicine, } \\
\text { Chongqing, } \\
\text { China } \\
\end{array}$ & $60 / 60$ & NR & XFZYD & Simvastatin & 1 month & $\begin{array}{l}\text { Clinical effect; TC; } \\
\text { TG; LDL-C; HDL- } \\
\text { C; adverse reaction }\end{array}$ \\
\hline Liu et al. & $\begin{array}{c}\text { The First } \\
\text { Affiliated } \\
\text { Hospital of } \\
\text { Heilongjiang } \\
\text { University of } \\
\text { Chinese } \\
\text { Medicine, } \\
\text { Heilongjiang } \\
\text { Province, China }\end{array}$ & $34 / 34$ & $2015.1-2016.1$ & $\begin{array}{c}\text { XFZYD + conventional } \\
\text { therapy }\end{array}$ & $\begin{array}{l}\text { Conventional } \\
\text { therapy }\end{array}$ & 1 month & $\begin{array}{l}\text { Clinical effect; } \\
\text { TCM syndrome } \\
\text { score }\end{array}$ \\
\hline Wang & $\begin{array}{c}\text { Nangong Ji Nan } \\
\text { Great Wall } \\
\text { Hospital, Hebei } \\
\text { Province, China } \\
\end{array}$ & $126 / 125$ & $2010.6-2012.6$ & $\begin{array}{c}\text { XFZYD + conventional } \\
\text { therapy }\end{array}$ & $\begin{array}{c}\text { Conventional } \\
\text { therapy }\end{array}$ & $\begin{array}{c}3 \\
\text { months }\end{array}$ & AF; DAP \\
\hline Wang & $\begin{array}{c}\text { The First } \\
\text { Affiliated } \\
\text { Hospital of } \\
\text { Henan } \\
\text { University of } \\
\text { Science and } \\
\text { Technology, } \\
\text { Henan } \\
\text { Province, China }\end{array}$ & $50 / 50$ & 2013.1-2014.1 & $\begin{array}{c}\text { XFZYD + conventional } \\
\text { therapy }\end{array}$ & $\begin{array}{c}\text { Conventional } \\
\text { therapy }\end{array}$ & $\begin{array}{c}3 \\
\text { months }\end{array}$ & Clinical effect \\
\hline $\begin{array}{l}\text { Wang } \\
\text { et al. }\end{array}$ & $\begin{array}{c}\text { Dagang } \\
\text { Hospital of } \\
\text { Traditional } \\
\text { Chinese } \\
\text { Medicine, } \\
\text { Binhai New } \\
\text { Area, Tianjin, } \\
\text { China } \\
\end{array}$ & $30 / 30$ & $2012.2-2013.7$ & $\begin{array}{c}\text { XFZYD + conventional } \\
\text { therapy }\end{array}$ & $\begin{array}{l}\text { Conventional } \\
\text { therapy }\end{array}$ & $\begin{array}{c}2 \\
\text { months }\end{array}$ & AF; DAP \\
\hline $\begin{array}{l}\text { Wang } \\
\text { et al. }\end{array}$ & $\begin{array}{c}\text { TeDa } \\
\text { Cardiovascular } \\
\text { Hospital and } \\
\text { Tianjin Chest } \\
\text { Hospital, } \\
\text { Tianjin, China }\end{array}$ & $75 / 75$ & 2011.1-2012.12 & $\begin{array}{c}\text { XFZYD + aspirin; } \\
\text { clopidogrel; } \\
\text { atorvastatin; metoprolol }\end{array}$ & $\begin{array}{l}\text { Aspirin; } \\
\text { clopidogrel; } \\
\text { atorvastatin; } \\
\text { metoprolol }\end{array}$ & 1 year & $\begin{array}{c}\text { Clinical effect; NO; } \\
\text { ET }\end{array}$ \\
\hline $\begin{array}{l}\text { Wang } \\
\text { et al. }\end{array}$ & $\begin{array}{l}\text { Dongzhimen } \\
\quad \text { Hospital, } \\
\text { Beijing, China } \\
\end{array}$ & $34 / 27$ & NR & $\begin{array}{c}\text { XFZYD + conventional } \\
\text { therapy }\end{array}$ & $\begin{array}{l}\text { Conventional } \\
\text { therapy }\end{array}$ & 1 month & $\begin{array}{c}\text { Clinical effect; } \\
\text { electrocardiogram } \\
\text { effect }\end{array}$ \\
\hline $\mathrm{Xi}$ and $\mathrm{Li}$ & $\begin{array}{c}\text { The First } \\
\text { Hospital of } \\
\text { Weinan City } \\
\text { and Xi'an } \\
\text { Yanliang } \\
\text { District } \\
\text { Hospital of } \\
\text { Traditional } \\
\text { Chinese } \\
\text { Medicine, } \\
\text { Shaanxi } \\
\text { Province, China }\end{array}$ & $20 / 20$ & 2018.9-2019.6 & XFZYD + metoprolol & Metoprolol & 1 month & Clinical effect \\
\hline
\end{tabular}


TABle 1: Continued.

\begin{tabular}{|c|c|c|c|c|c|c|c|}
\hline Author(s) & $\begin{array}{l}\text { Country and } \\
\text { setting }\end{array}$ & $\begin{array}{c}\text { Sample size } \\
\text { (experimental/ } \\
\text { control) }\end{array}$ & $\begin{array}{l}\text { Patient } \\
\text { enrollment time }\end{array}$ & Experimental & Control & Duration & Outcome measures \\
\hline $\begin{array}{l}\text { Yang and } \\
\text { Zhang }\end{array}$ & $\begin{array}{l}\text { Yicheng Central } \\
\text { Health Center } \\
\text { in Zaozhuang } \\
\text { City, Shandong } \\
\text { Province, China }\end{array}$ & $75 / 75$ & $2015.3-2017.3$ & $\begin{array}{c}\text { XFZYD + conventional } \\
\text { therapy }\end{array}$ & $\begin{array}{l}\text { Conventional } \\
\text { therapy }\end{array}$ & 1 month & Clinical effect \\
\hline $\begin{array}{l}\text { Yang } \\
\text { et al. }\end{array}$ & $\begin{array}{c}\text { Hebei Tangshan } \\
\text { Workers' } \\
\text { Hospital, Hebei } \\
\text { Province, China } \\
\end{array}$ & $48 / 48$ & 2013.12-2014.12 & $\begin{array}{c}\text { XFZYD + aspirin; } \\
\text { isosorbide mononitrate }\end{array}$ & $\begin{array}{l}\text { Aspirin; } \\
\text { isosorbide } \\
\text { mononitrate }\end{array}$ & 1 month & $\begin{array}{l}\text { Clinical effect; AF; } \\
\text { DAP; TC; TG; } \\
\text { LDL-C; HDL-C; } \\
\text { adverse reaction }\end{array}$ \\
\hline Yuan & $\begin{array}{c}\text { Guangzhou } \\
\text { Hospital of } \\
\text { Traditional } \\
\text { Chinese } \\
\text { Medicine, } \\
\text { Guangdong } \\
\text { Province, China } \\
\end{array}$ & $30 / 30$ & $2015.3-2016.3$ & $\begin{array}{c}\text { XFZYD + conventional } \\
\text { therapy }\end{array}$ & $\begin{array}{l}\text { Conventional } \\
\text { therapy }\end{array}$ & 14 days & $\begin{array}{l}\text { Clinical effect; } \\
\text { TCM syndrome } \\
\text { score }\end{array}$ \\
\hline Zhang & $\begin{array}{c}\text { The First } \\
\text { Affiliated } \\
\text { Hospital of } \\
\text { Guangzhou } \\
\text { University of } \\
\text { Chinese } \\
\text { Medicine, } \\
\text { Guangdong } \\
\text { Province, China }\end{array}$ & $30 / 30$ & NR & XFZYD & $\begin{array}{l}\text { Aspirin; } \\
\text { atorvastatin; } \\
\text { perindopril; } \\
\text { metoprolol; }\end{array}$ & 3 weeks & $\begin{array}{l}\text { Clinical effect; AF; } \\
\text { DAP; TCM } \\
\text { syndrome score }\end{array}$ \\
\hline Zhang & $\begin{array}{c}\text { Zhengzhou } \\
\text { People's } \\
\text { Hospital, Henan } \\
\text { Province, China }\end{array}$ & $50 / 50$ & 2008.1-2011.12 & $\begin{array}{c}\text { XFZYD + aspirin; } \\
\text { isosorbide mononitrate; } \\
\text { metoprolol }\end{array}$ & $\begin{array}{l}\text { Aspirin; } \\
\text { isosorbide } \\
\text { mononitrate; } \\
\text { metoprolol }\end{array}$ & 1 month & $\begin{array}{c}\text { Clinical effect; } \\
\text { electrocardiogram } \\
\text { effect; TCM } \\
\text { syndrome score; } \\
\text { TC; LDL-C; HDL- } \\
\text { C; }\end{array}$ \\
\hline Zhang & $\begin{array}{c}\text { Traditional } \\
\text { Chinese } \\
\text { Medicine of } \\
\text { Ruzhou City, } \\
\text { Henan } \\
\text { Province, China }\end{array}$ & $52 / 52$ & 2014.1-2016.1 & $\begin{array}{c}\mathrm{XFZYD}+\text { conventional } \\
\text { therapy }\end{array}$ & $\begin{array}{l}\text { Conventional } \\
\text { therapy }\end{array}$ & $\begin{array}{c}2 \\
\text { months }\end{array}$ & $\begin{array}{l}\text { Clinical effect; AF; } \\
\text { DAP; TC; HDL-C }\end{array}$ \\
\hline Zhang & $\begin{array}{c}\text { Songzi Hospital } \\
\text { of Traditional } \\
\text { Chinese } \\
\text { Medicine, } \\
\text { Hubei Province, } \\
\text { China }\end{array}$ & $73 / 73$ & $2017.12-2019.2$ & $\begin{array}{l}\mathrm{XFZYD}+\text { aspirin; } \\
\text { isosorbide nitrate }\end{array}$ & $\begin{array}{l}\text { Aspirin; } \\
\text { isosorbide } \\
\text { nitrate }\end{array}$ & 28 days & $\begin{array}{c}\text { TCM syndrome } \\
\text { score; WBV; PV; } \\
\text { FB }\end{array}$ \\
\hline $\begin{array}{l}\text { Zhang } \\
\text { and } \\
\text { Wang }\end{array}$ & $\begin{array}{c}\text { Minquan } \\
\text { County } \\
\text { Hospital of } \\
\text { Traditional } \\
\text { Chinese } \\
\text { Medicine and } \\
\text { The First } \\
\text { Affiliated } \\
\text { Hospital of } \\
\text { Henan College } \\
\text { of Traditional } \\
\text { Chinese } \\
\text { Medicine, } \\
\text { Henan } \\
\text { Province, China }\end{array}$ & $20 / 20$ & NR & $\begin{array}{l}\text { XFZYD + aspirin; low } \\
\text { molecular heparin; } \\
\text { nitroglycerin; } \\
\text { metoprolol; valsartan; } \\
\text { nifedipine }\end{array}$ & $\begin{array}{l}\text { Aspirin; low } \\
\text { molecular } \\
\text { heparin; } \\
\text { nitroglycerin; } \\
\text { metoprolol; } \\
\text { valsartan; } \\
\text { nifedipine }\end{array}$ & 1 month & $\begin{array}{c}\text { Clinical effect; } \\
\text { electrocardiogram } \\
\text { effect }\end{array}$ \\
\hline
\end{tabular}


TABle 1: Continued.

\begin{tabular}{|c|c|c|c|c|c|c|c|}
\hline Author(s) & $\begin{array}{l}\text { Country and } \\
\text { setting }\end{array}$ & $\begin{array}{l}\text { Sample size } \\
\text { (experimental/ } \\
\text { control) }\end{array}$ & $\begin{array}{c}\text { Patient } \\
\text { enrollment time }\end{array}$ & Experimental & Control & Duration & Outcome measures \\
\hline $\begin{array}{l}\text { Zhang } \\
\text { et al. }\end{array}$ & $\begin{array}{c}\text { Luyi County } \\
\text { People's } \\
\text { Hospital, Henan } \\
\text { Province, China }\end{array}$ & $55 / 50$ & 2015.9-2018.9 & $\begin{array}{c}\text { XFZYD + aspirin; } \\
\text { isosorbide mononitrate; } \\
\text { metoprolol; } \\
\text { nitroglycerin }\end{array}$ & $\begin{array}{c}\text { Aspirin; } \\
\text { isosorbide } \\
\text { mononitrate; } \\
\text { metoprolol; } \\
\text { nitroglycerin }\end{array}$ & 14 days & $\begin{array}{l}\text { Clinical effect; } \\
\text { electrocardiogram } \\
\text { effect; AF; DAP; } \\
\text { WBV; PV; FB }\end{array}$ \\
\hline Zheng & $\begin{array}{c}\text { Nanping } \\
\text { Second } \\
\text { Hospital, Fujian } \\
\text { Province, China }\end{array}$ & $46 / 46$ & 2016.1-2017.10 & XFZYD + atorvastatin & Atorvastatin & 1 month & $\begin{array}{c}\text { AF; DAP; TC; TG; } \\
\text { ldl-c; hdl-c; WBV; } \\
\text { PV; FB }\end{array}$ \\
\hline
\end{tabular}

AF: angina frequency; DAP: duration of angina pectoris; ET: endothelin; FB: fibrinogen; HDL-C: high-density lipoprotein cholesterol; LDL-C: low-density lipoprotein cholesterol; NR: not reported; PV: plasma viscosity; TC: total cholesterol; TG: triglyceride; WBV: whole blood viscosity; XFZYD: Xue-Fu-Zhu-Yu decoction.

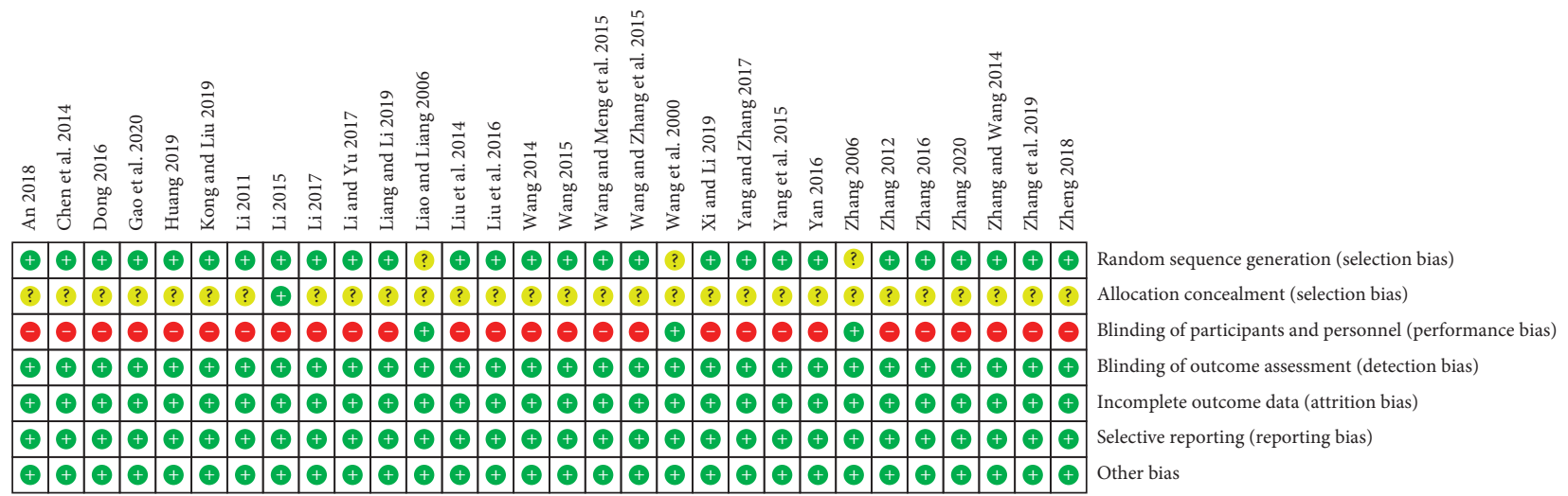

FIGURE 2: Methodological quality assessment results for all included trials.

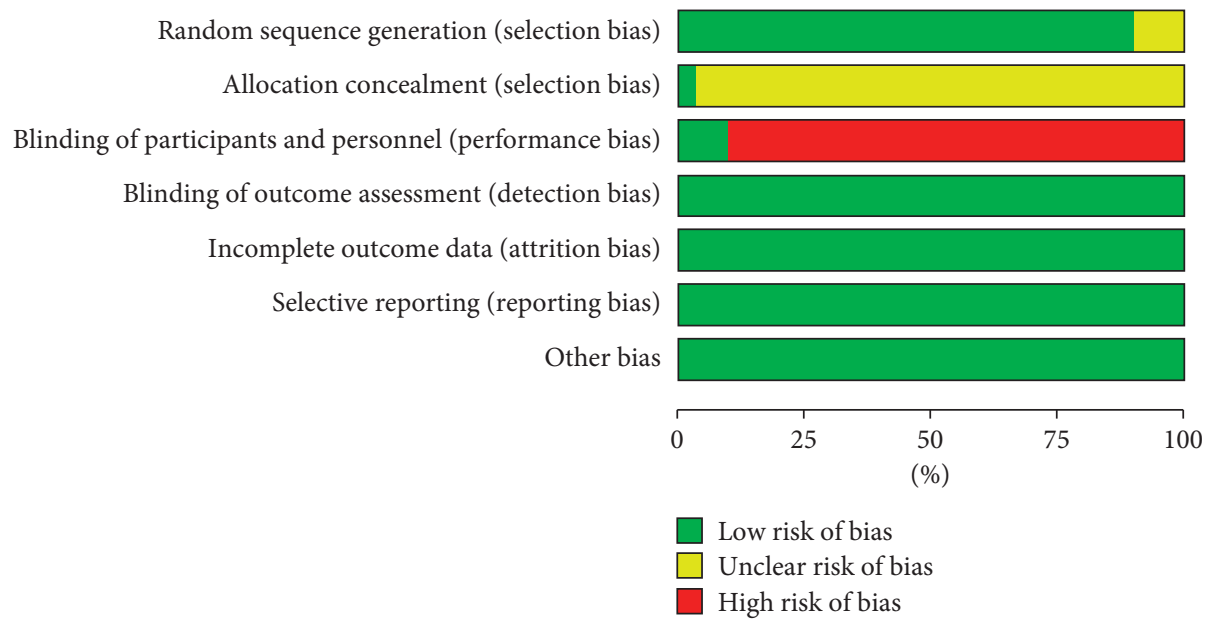

Figure 3: Percentage results of risk of bias for each of the included trials.

pain of patients when compared with CD ( 8 trials; $n=1259$; $\mathrm{MD},-1.39$; $95 \% \mathrm{CI},-1.98$ to -0.80$)$.

3.4.2. Clinical Effect. The clinical effect was established with reference to the Standard of Diagnosis and Efficacy of Diseases in traditional Chinese Medicine, and the clinical effect standard was divided into cure, apparent efficacy, effectiveness, and ineffectiveness to evaluate the clinical effect of using XFZYD. The study treated patients with CHD, and 22 trials have been reported. In addition, meta-analysis revealed that XFZYD could significantly improve the clinical effect of CHD, and its effect was 1.24 times higher than that of CD (22 trials; $n=2089$; RR; $1.24 ; 95 \%$ CI, 1.19 to 1.29 ; 


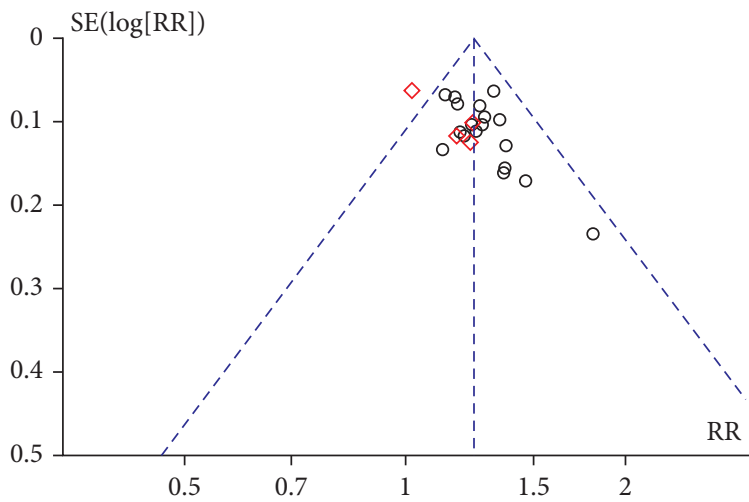

Subgroups
$\circ$ XCWC vs. CD
$\diamond$ XFZYD vs. CD

FIGURE 4: Inverted funnel plot of XFZYD in the treatment of CHD for clinical effect.

\begin{tabular}{|c|c|c|c|c|c|c|c|c|c|c|c|c|}
\hline \multirow{3}{*}{$\begin{array}{l}\text { Study or subgroup } \\
\text { Li } 2011\end{array}$} & \multicolumn{3}{|c|}{ XCWC } & \multicolumn{3}{|c|}{$\mathrm{CD}$} & \multirow{2}{*}{$\begin{array}{l}\text { Weight } \\
(\%)\end{array}$} & \multirow{2}{*}{$\begin{array}{l}\text { Mean Difference } \\
\text { IV, Random, 95\% CI }\end{array}$} & \multirow{2}{*}{\multicolumn{4}{|c|}{$\begin{array}{c}\text { Mean Difference } \\
\text { IV, Random, 95\% CI }\end{array}$}} \\
\hline & Mean & SD & Total & Mean & SD & Total & & & & & & \\
\hline & 0.83 & 0.21 & 45 & 2.07 & 0.31 & 45 & 11.2 & $-1.24[-1.35,-1.13]$ & -- & & & \\
\hline Li 2015 & 2 & 0.7 & 126 & 3.2 & 0.8 & 125 & 10.9 & $-1.20[-1.39,-1.01]$ & $\longrightarrow$ & & & \\
\hline Liang and Li 2019 & 2.13 & 0.55 & 150 & 3.27 & 0.71 & 150 & 11.1 & $-1.14[-1.28,-1.00]$ & -- & & & \\
\hline Wang 2014 & 2 & 0.7 & 126 & 3.2 & 0.8 & 125 & 10.9 & $-1.20[-1.39,-1.01]$ & - - & & & \\
\hline Wang and Meng et al. 2015 & 1.2 & 0.1 & 30 & 1.5 & 0.3 & 30 & 11.2 & $-0.30[-0.41,-0.19]$ & & -- & & \\
\hline Yang et al. 2015 & 0.97 & 0.1 & 48 & 1.6 & 0.22 & 48 & 11.3 & $-0.63[-0.70,-0.56]$ & & - & & \\
\hline Zhang 2016 & 1 & 0.2 & 52 & 1.8 & 0.4 & 52 & 11.2 & $-0.80[-0.92,-0.68]$ & & & & \\
\hline Zhang et al. 2019 & 1.12 & 0.48 & 55 & 3.12 & 0.36 & 50 & 11.0 & $-2.00[-2.16,-1.84]$ & & & & \\
\hline Zheng 2018 & 0.61 & 0.23 & 46 & 1.22 & 0.33 & 46 & 11.2 & $-0.61[-0.73,-0.49]$ & & -- & & \\
\hline \multirow{2}{*}{\multicolumn{12}{|c|}{$\begin{array}{l}\text { Total }(95 \% \mathrm{CI}) \\
\text { Heterogeneity: } \mathrm{Tau}^{2}=0.20, \mathrm{Chi}^{2}=434.24, \mathrm{df}=8(\mathrm{P}<0.00001) ; \mathrm{I}^{2}=98 \% \\
\text { Test for overall effect: } \mathrm{Z}=6.66(\mathrm{P}<0.00001)\end{array}$}} & \\
\hline & & & & & & & & & & & & \\
\hline & & & & & & & & & -1 & -0.5 & 0.5 & 1 \\
\hline
\end{tabular}

(a)

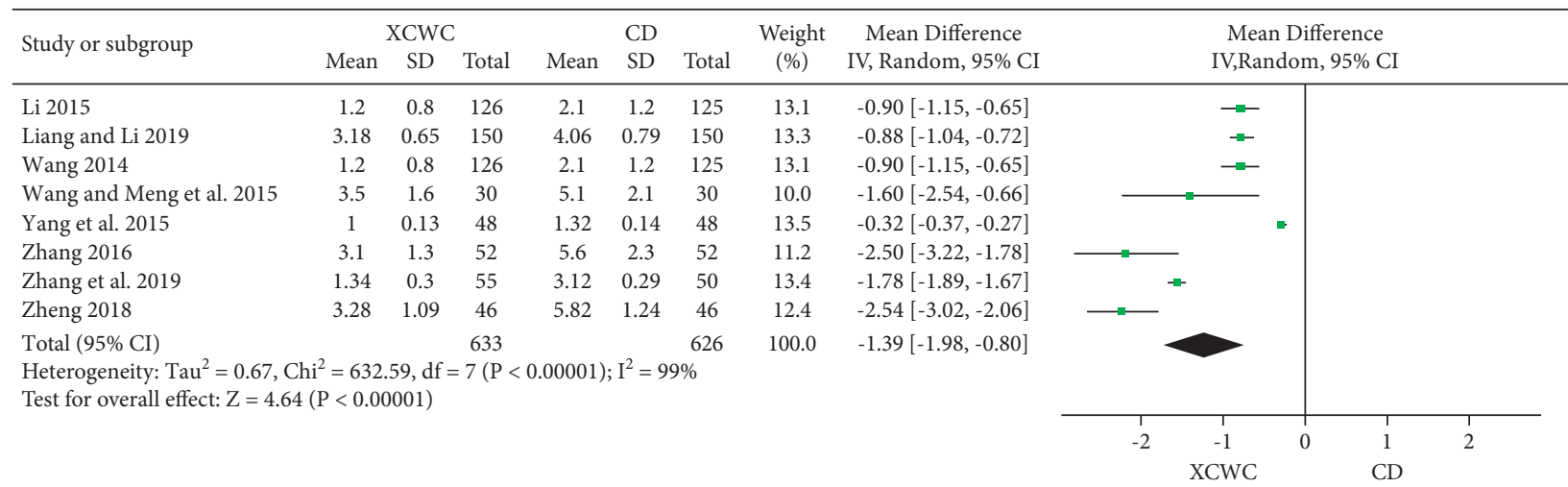

(b)

Figure 5: Forest plot of the trials showing angina in XCWC vs. CD: (a) AF; (b) DAP.

Figure 6). Among them, 18 trials [11, 13-18, 22, 24-26, $28,30-32,35,36,40]$ treated participants with XCWC and $\mathrm{CD}$ in experimental group and control group, respectively, and 4 trials [21, 33, 38, 39] used XFZYD and CD. Metaanalysis identified that XFZYD could observably enhance the clinical efficacy whether used alone or in combination with CD, which were 1.13 ( 4 trials; $n=320$; RR; 1.13 ; 95\% CI, 1.03 to 1.24 ) and 1.26 (18 trials; $n=1769$; RR; 1.26 ; $95 \% \mathrm{CI}$, 1.20 to 1.32 ) times higher than those of $\mathrm{CD}$, respectively.
3.4.3. Electrocardiogram Effect. The electrocardiogram effect was reported in 7 trials $[17,32,35-38,40]$, which was judged according to the changes of electrocardiogram before and after treatment. XFZYD showed an obvious improvement on electrocardiogram, and its effect was 1.31 times higher than that of CD (7 trials; $n=619$; RR; $1.31 ; 95 \% \mathrm{CI}$, 1.18 to 1.46 ; Figure 7 ), which identified that XFZYD could significantly alleviate myocardial ischemia in patients. Additionally, the effect of XFZYD was 1.63 ( 1 trial; $n=679$; 


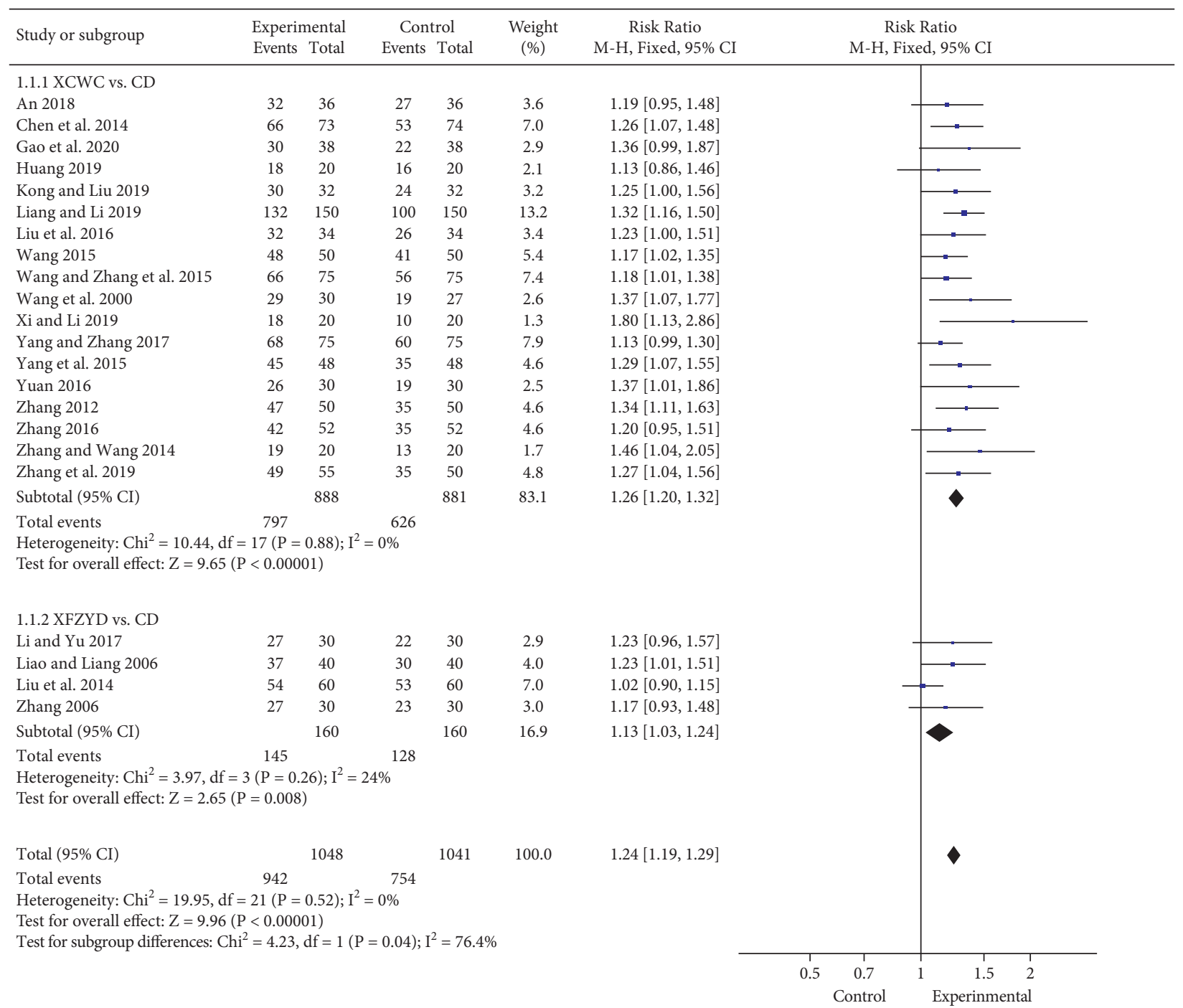

FIgURE 6: Forest plot of the trials showing clinical effect in different interventions.

RR; 1.63 ; $95 \%$ CI, 1.04 to 2.53 ) times higher than that of CD. Meta-analysis of 6 trials [17, 31, 35-37, 40] comparing XCWC with CD revealed a remarkable increasing effect of XCWC in electrocardiogram effect, which was 1.28 times higher than that of CD (6 trials; $n=539$; RR; $1.28 ; 95 \% \mathrm{CI}$, 1.15 to 1.43$)$.

3.4.4. Blood Lipids (TC, TG, LDL-C, and HDL-C). The levels of TC and TG at baseline and after intervention were reported in $9[13,19,23,26,31-33,36,37]$ and 6 $[13,19,31-33,37]$ trials, respectively, and the forest plots for the meta-analysis of each relevant drug are provided in Figures 8(a) and 8(b). Compared with CD, TC (7 trials; $n=659 ; \mathrm{MD},-0.91 ; 95 \% \mathrm{CI},-1.28$ to -0.53$)$ and TG (4 trials; $n=375 ; \mathrm{MD},-0.46 ; 95 \% \mathrm{CI},-0.64$ to -0.27$)$ were both significantly lower in the XCWC group. However, XFZYD did not show obvious advantages in TC ( 2 trials; $n=200$; $\mathrm{MD},-0.23$; $95 \% \mathrm{CI},-1.34$ to 0.88$)$ and TG (2 trials; $n=200$; $\mathrm{MD},-0.34 ; 95 \% \mathrm{CI},-2.23$ to 1.55$)$.
Eight trials $[13,19,23,31-33,36,38]$ reported the levels of LDL-C at baseline and after treatment. The higher the LDL-C, the higher the risk of CHD. Compared with CD, LDL-C was remarkably lower in the XCWC group (6 trials; $n=555 ; \mathrm{MD},-0.67 ; 95 \% \mathrm{CI},-0.94$ to -0.39 ; Figure $8(\mathrm{c})$ ). However, there was no obvious difference between XFZYD and CD (2 trials; $n=200$; MD, $0.06 ; 95 \% \mathrm{CI},-0.58$ to 0.71 ).

$\mathrm{HDL}-\mathrm{C}$ is a protective factor of $\mathrm{CHD}$ and can resist atherosclerosis, which was reported in 8 trials $[13,19,26,31-33,36,38]$. In the meta-analysis, XCWC exhibited a significantly greater increase in HDL-C than that of CD alone ( 6 trials; $n=579 ; \mathrm{MD}, 0.32 ; 95 \% \mathrm{CI}, 0.16$ to 0.47 ; Figure $8(\mathrm{~d}))$, but XFZYD did not show remarkable advantages ( 2 trials; $n=200 ; \mathrm{MD}, 0.12 ; 95 \%$ CI, -0.29 to 0.53 ).

3.4.5. Hemorheology Index ( $W B V, P V$, and $F B)$. Three trials $[12,17,19]$ reported the level of hemorheology index at baseline and after intervention. Meta-analysis evaluated that 


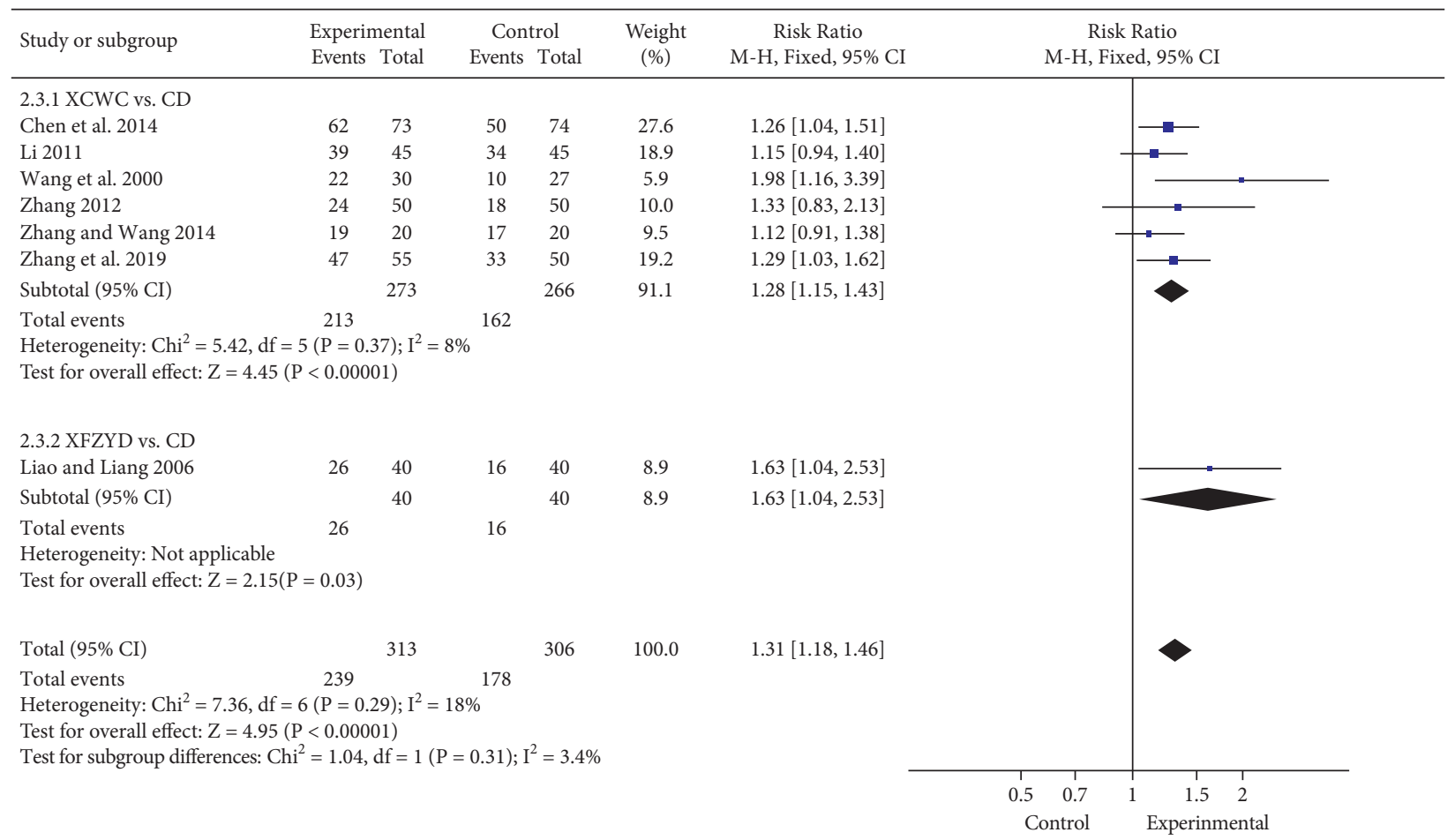

FIGURE 7: Forest plot of the trials showing electrocardiogram effect in different interventions.

the combination of XCWC and CD could significantly decrease hemorheology index to reduce blood viscosity (MD, $-0.58 ; 95 \% \mathrm{CI},-0.69$ to -0.46 ; Figure 9 ), which not only decreased WBV ( 2 trials; $n=238 ; \mathrm{MD},-0.73 ; 95 \% \mathrm{CI}$, -0.96 to -0.50 ) and PV ( 3 trials; $n=343$; MD, -0.46 ; $95 \% \mathrm{CI}$, -0.65 to -0.28$)$ but also exhibited a great advantage in reducing $\mathrm{FB}$ level ( 3 trials; $n=343$; MD,$-0.65 ; 95 \% \mathrm{CI},-0.79$ to -0.52$)$.

3.4.6. Vascular Endothelial Function Indicators (NO and ET). Three trials $[11,30,39]$ reported the levels of NO at baseline and after treatment. Compared with $\mathrm{CD}, \mathrm{NO}$ level was remarkably increased in the XFZYD ( 1 trial; $n=60$; MD, 6.50; 95\% CI, 0.56 to 12.44; Figure 10(a)) and XCWC (2 trials; $n=226$; MD , 4.68; $95 \%$ CI, 4.23 to 5.12; Figure $10(\mathrm{a})$ ) group.

The effect of XFZYD on ET level was reported in the same trials as NO. Compared with $\mathrm{CD}$, significant lowering effects on ET level by XFZYD alone ( 1 trial; $n=60$; MD, -15.82 ; $95 \% \mathrm{CI},-23.24$ to -8.4 ; Figure $10(\mathrm{~b}))$ or in combination with $\mathrm{CD}(2$ trials; $n=226$; MD, $-13.69 ; 95 \% \mathrm{CI}$, -17.75 to -9.62 ; Figure 10 (b)) were both identified.

3.4.7. TCM Syndrome Score. Four trials reported TCM syndrome score $[12,24,25,39]$. The scoring method refers to the guiding principles for clinical research of new Chinese medicine [41], and scores are zero, one, two, and three points, respectively, according to the degree of clinical manifestations. The lower the score, the better the effect. Meta-analysis revealed that XFZYD could obviously reduce TCM syndrome score compared with CD ( 1 trial; $n=60$;
$\mathrm{MD},-2.07 ; 95 \% \mathrm{CI},-3.61$ to -0.53 ; Figure 11$)$, but it did not show remarkable difference when combined with CD (3 trials; $n=274$; MD, $-5.12 ; 95 \% \mathrm{CI},-10.60$ to 0.35 ).

3.4.8. Adverse Reaction. Six trials $[15,20,21,31,33,38]$ reported adverse reactions of the experimental group and control group after treatment, including headache, nausea, vomiting, diarrhea, extrasystole, hypotension, myocardial infarction, and elevated transaminase. However, when combined with CD, XFZYD alone ( 3 trials; $n=260$; RR, 0.78; 95\% CI, 0.20 to 3.10; Figure 12) or in combination with CD (3 trials; $n=456$; RR, 0.62 ; $95 \% \mathrm{CI}, 0.35$ to 1.12 ; Figure 12 ) did not show obvious advantages.

\section{Discussion}

Among the 3 previous reviews, 2 were protocols $[5,6]$, and only 1 paper was the final study [7], which studied the efficacy of Xue-Fu-Zhu-Yu capsule in the treatment of unstable angina pectoris, and a total of 8 trials were included. It only had 4 outcomes including the incidence of a heart event, reduction of angina symptoms, ECG improvement, and quality of life. Our study was more optimized in terms of strict standards, rigorous screening process, multiple included trials, and comprehensive outcomes, and the results were more reliable, which provided help for clinical drug use from many aspects and filled the gap of precision medicine. In this study, both the number of included trials and the outcome measures were increased significantly. We comprehensively analyzed the efficacy and safety of XFZYD for CHD, explored the potential mechanism of XFZYD treatment from aspects of ECG improvement, blood lipid 


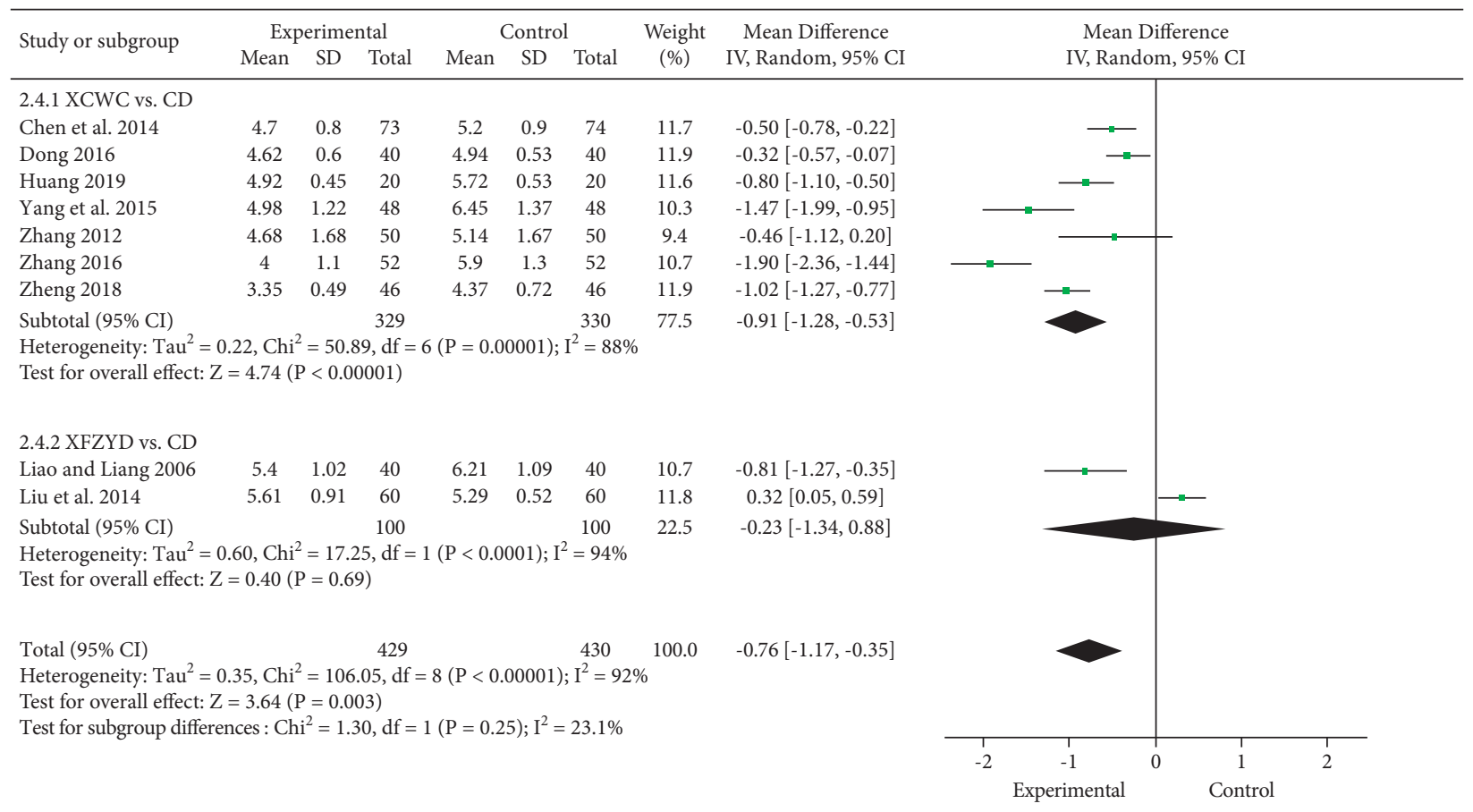

(a)

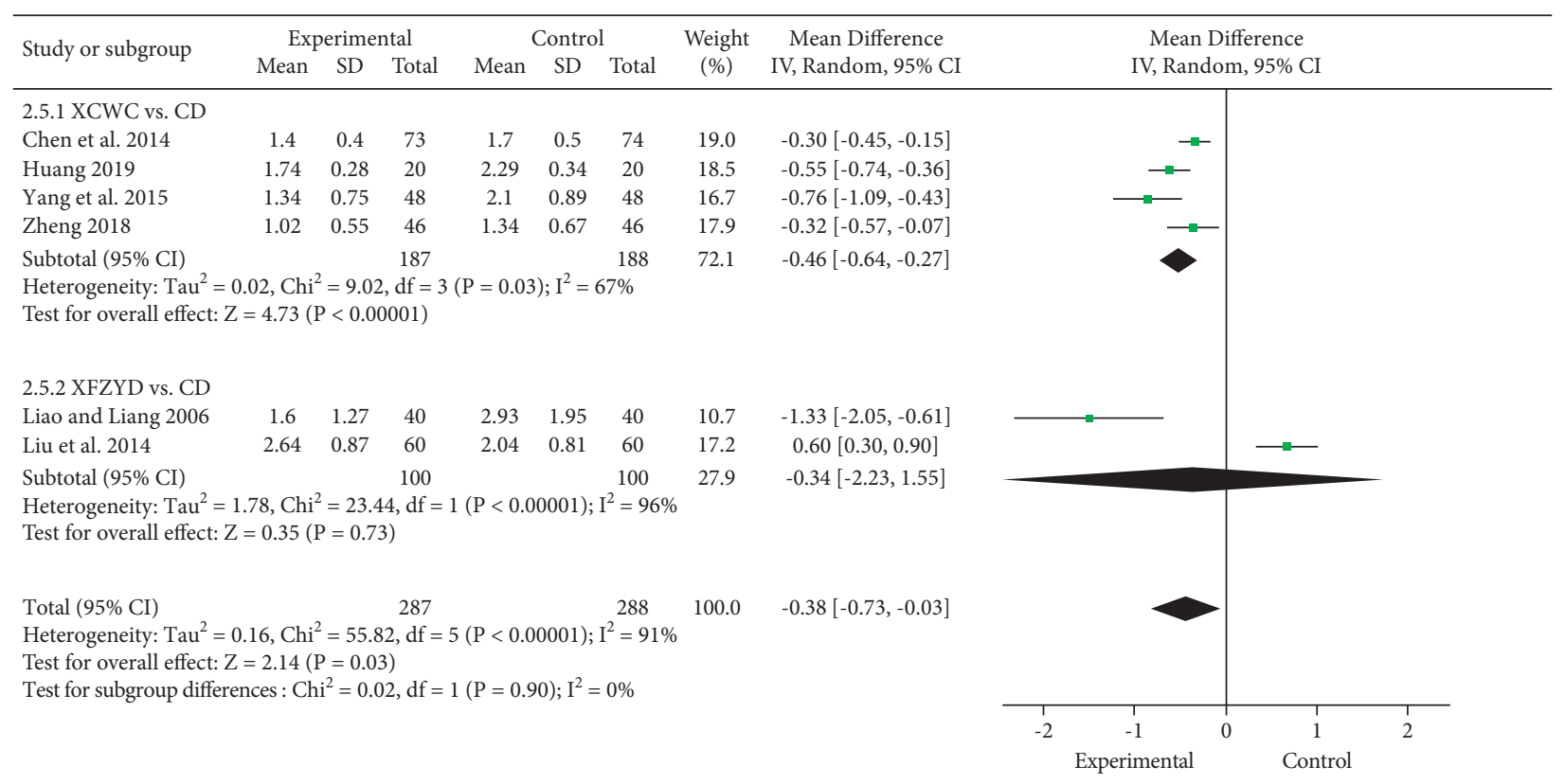

(b)

Figure 8: Continued. 


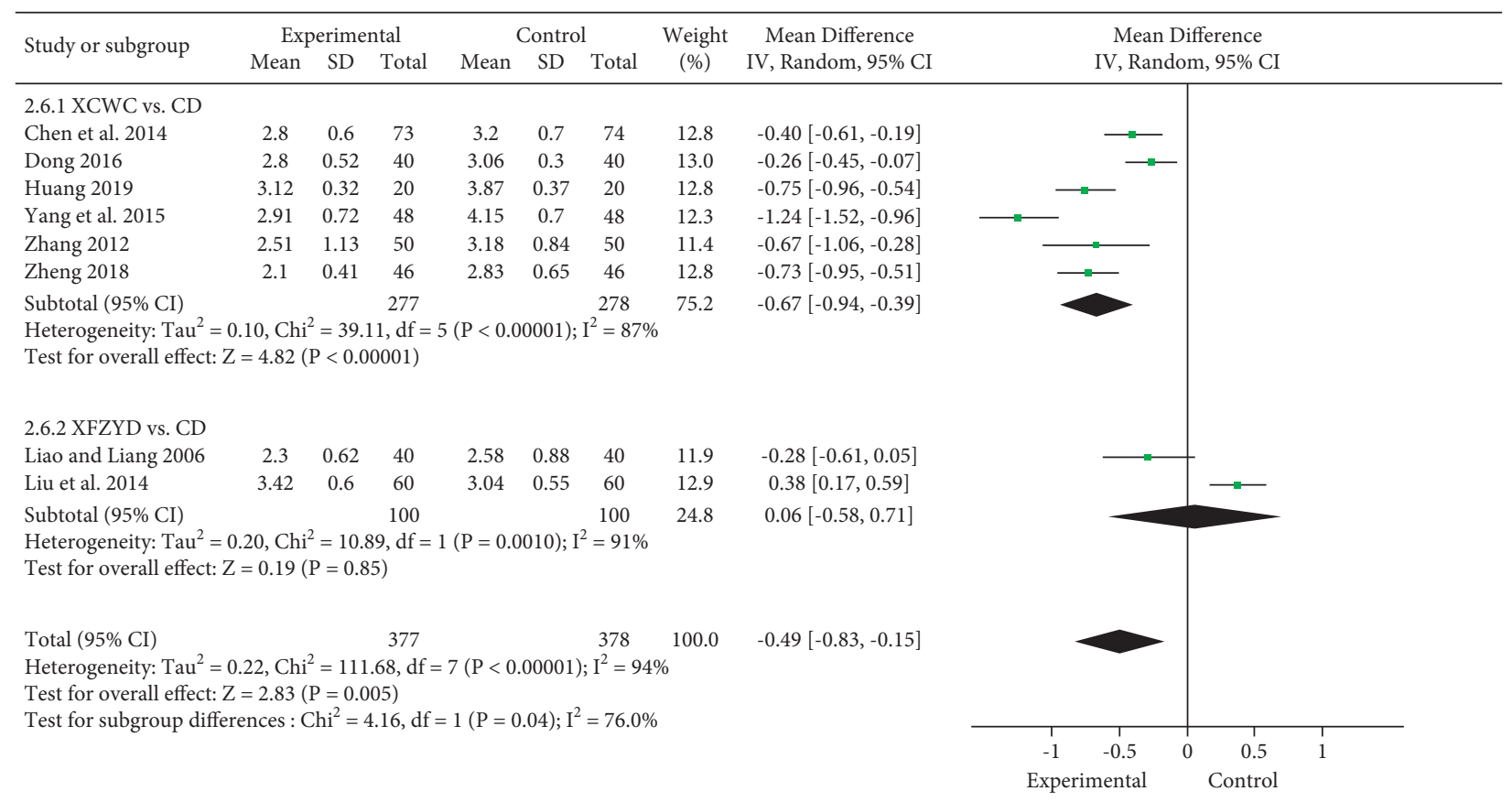

(c)

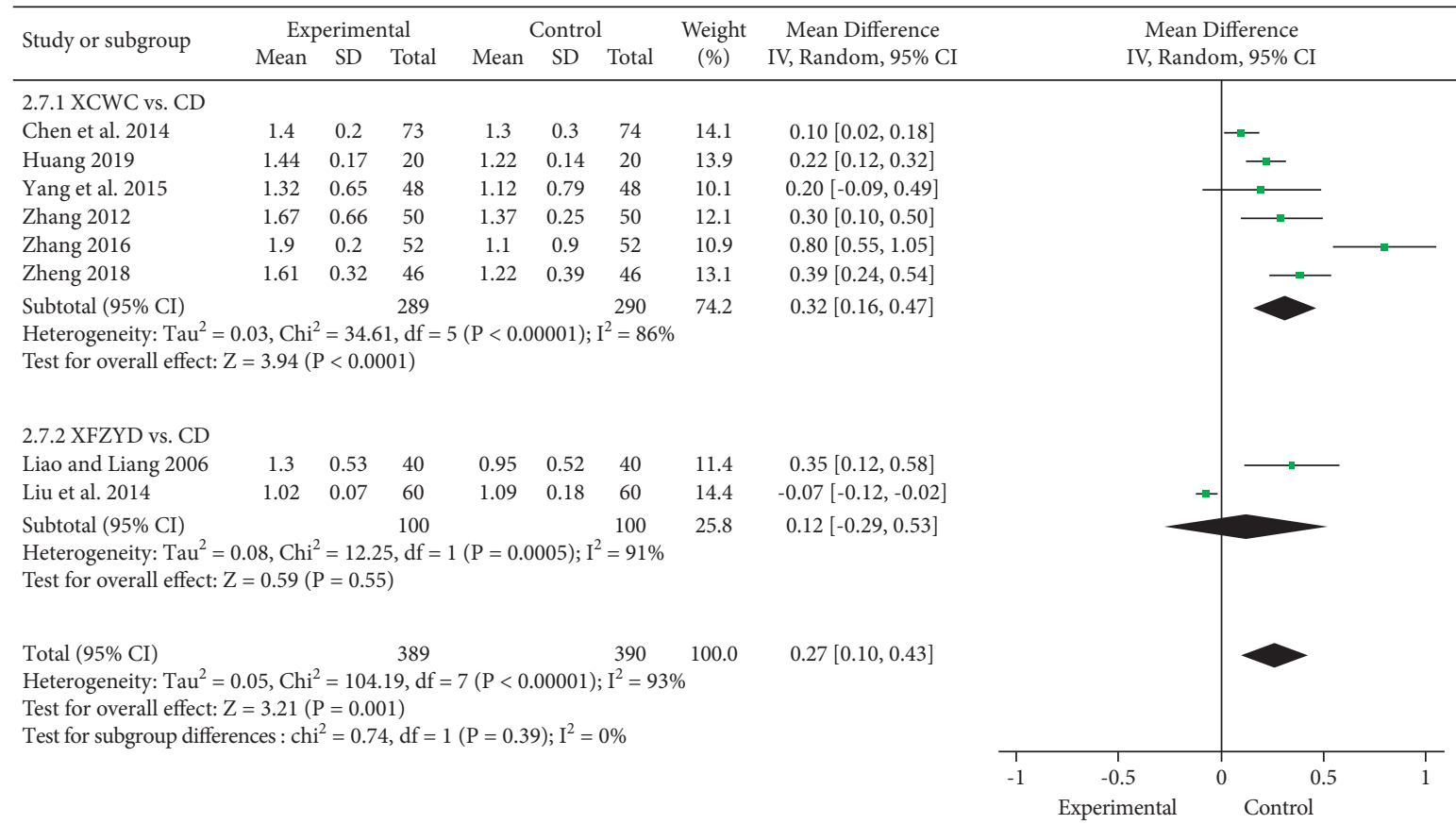

(d)

FIGURE 8: Forest plot of the trials showing blood lipids in different interventions: (a) TC; (b) TG; (c) LDL-C; (d) HDL-C.

improvement, hemorheology, and vascular endothelial function, and then compared TCM syndrome scores of patients before and after treatment to judge the improvement of syndrome and life quality of patients. And the safety of XFZYD was evaluated according to the occurrence of adverse reactions after taking the drug.

The efficacy and safety of XFZYD for CHD were evaluated by meta-analysis on the basis of 30 trials and 3126 participants. The results showed that XFZYD alone or in combination with $\mathrm{CD}$ could significantly improve the clinical effect at 1.13 and 1.26 times higher than that of $C D$ alone. At the same time, XFZYD could also obviously improve the curative effect of electrocardiogram and the condition of myocardial ischemia in patients, which was 1.63 times that of CD. Using XFZYD alone could not only reduce DAP, increase the level of NO, and lower the level of ET to improve vascular endothelial function, but also remarkably decrease TCM syndrome score and alleviate the symptoms 


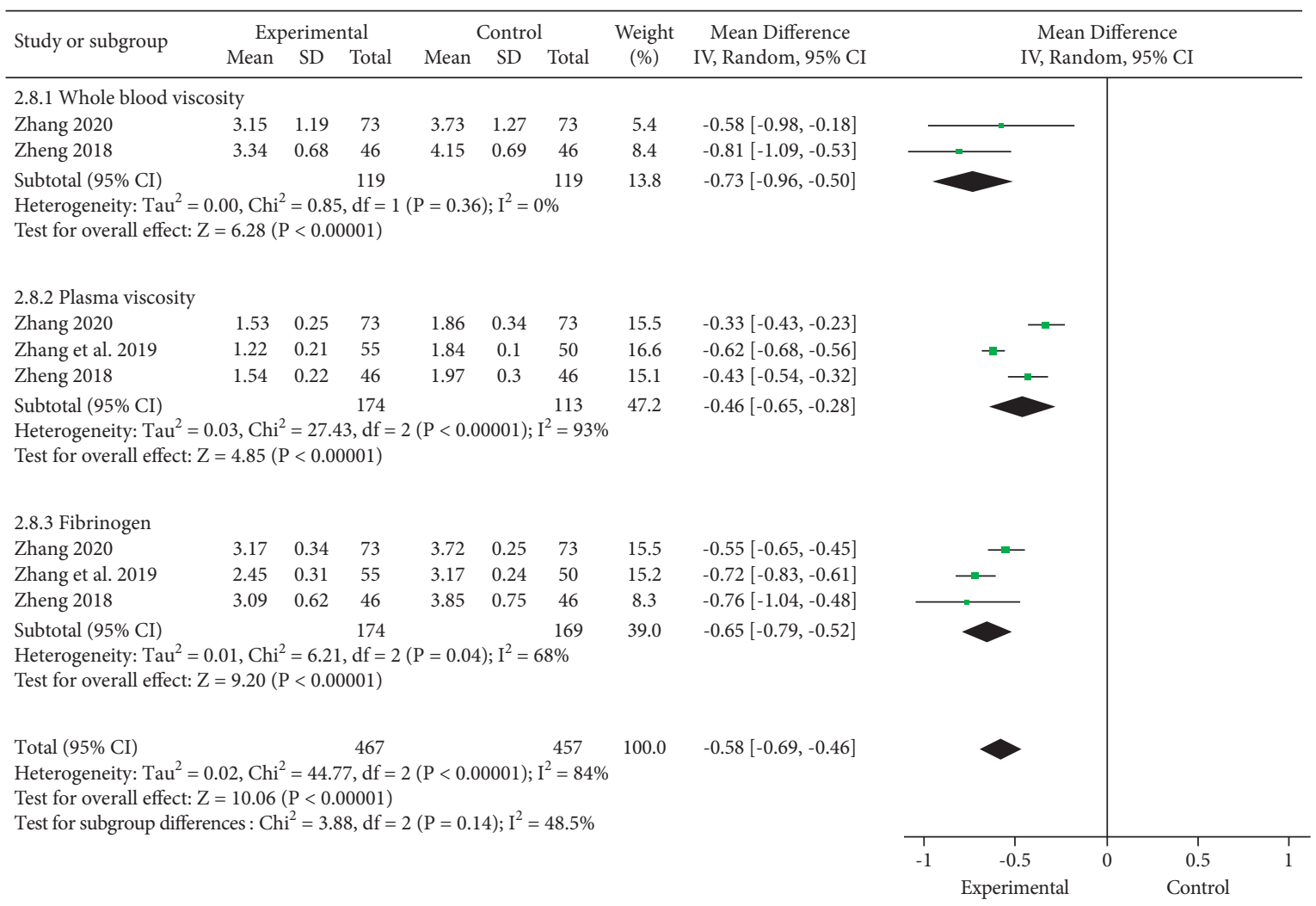

FIGURE 9: Forest plot of the trials showing hemorheology index.

of patients. Comparing with XFZYD alone, its combination with $\mathrm{CD}$ could not only reduce AF to relieve the pain of patients with CHD, decrease the levels of TC, TG, and LDL$\mathrm{C}$, and increase HDL-C, thereby correcting the dyslipidemia, but also lower the blood viscosity by decreasing the levels of WBC, PV, and FB. Therefore, XFZYD can treat CHD through multitarget comprehensive intervention. This study is the first systematic review and meta-analysis with some unique ways, including its comprehensiveness, large-scale search, the novelty of findings, and transparent approach, which provided a strong evidence support for the accurate use of medicine for CHD in clinics.

XFZYD can demonstrate its unique advantages in the treatment of CHD through the multitarget comprehensive action of multiple drugs. Taoren and Honghua promote blood circulation to arrest pain, Chishao and Chuanxiong promote blood circulation for removing blood stasis, Niuxi activates blood to promote menstruation and ensures proper downward flow of the blood, Shengdi and Danggui nourish Yin and blood, Jiegeng and Zhiqiao promote the circulation of Qi, Chaihu disperses stagnated hepatoqi, and Gancao coordinates the drug actions of a prescription. So, XFZYD can activate blood stasis and dissipate Qi, and then all symptoms can be cured, which is a good TCM formula for treating blood stasis. Modern pharmacological researches have shown that Taoren had antithrombotic, anticoagulant, and lipid-lowering effects, which could inhibit atherosclerotic plaque formation, prevent myocardial infarction, and improve hemodynamics [42]. Hydroxysafflor yellow A, one main bioactive component of Honghua, may inhibit coronary artery endothelial cell damage by increasing NO expression and release and hinder platelet aggregation, which could play an antiatherosclerotic effect [43]. The combination of Taoren and Honghua could not only significantly reduce blood viscosity through decreasing the concentration of FB and improving the aggregation of red blood cells and platelets, but also participate in various biological processes and signal pathways, as well as interfering with the occurrence and development of CHD, which played a protective and repairing effect on the cardiovascular system. Chuanxiong had the effects of antiatherosclerosis, lipid-lowering, dilating blood vessels, antiplatelet aggregation, antithrombosis, and so on [44]. The peony total glycosides of Chishao had the effects of antiatherosclerosis and stabilizing plaque through lipid-lowering, anti-inflammatory, and inhibiting angiogenesis [45], which could also obviously improve hemorheology, protect vascular endothelial function, and play a role in antiatherosclerosis; furthermore, it could improve myocardial ischemia when used with Chuanxiong [46]. Niuxi could not only reduce WBV and red blood cell aggregation index, but also prolong prothrombin time. Shengdi could lower blood lipids [47], protect the cardiovascular system, improve cell function, and avoid ischemic injury [48]. Danggui could decrease vascular resistance, reduce thrombosis, and improve blood circulation [49], thereby reducing blood lipids and atherosclerosis. Jiegeng could resist oxidation, lower blood lipids, and protect myocardium [50]. Naringin of Zhiqiao could not only reduce TC through multiple mechanisms, but also inhibit platelet aggregation [51], and hesperetin also had the 


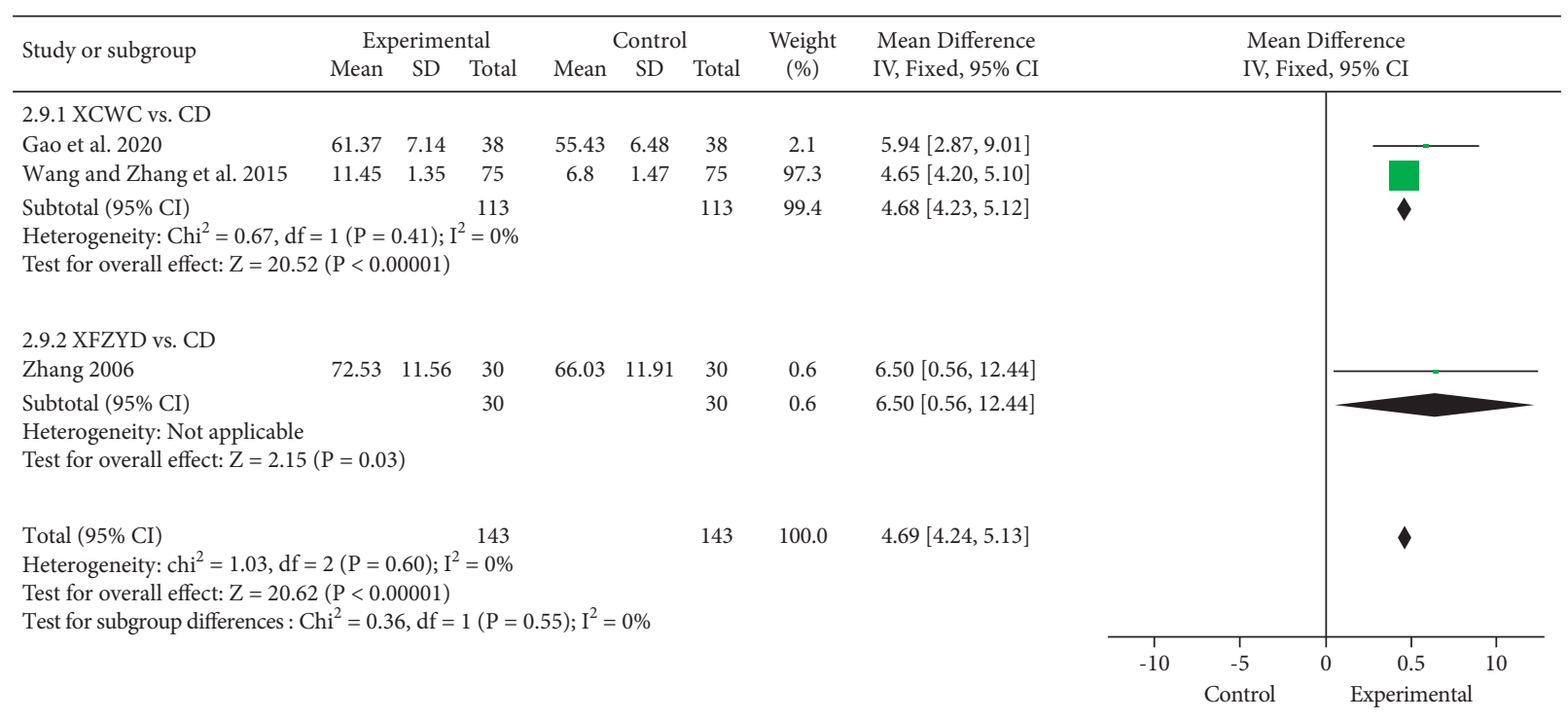

(a)

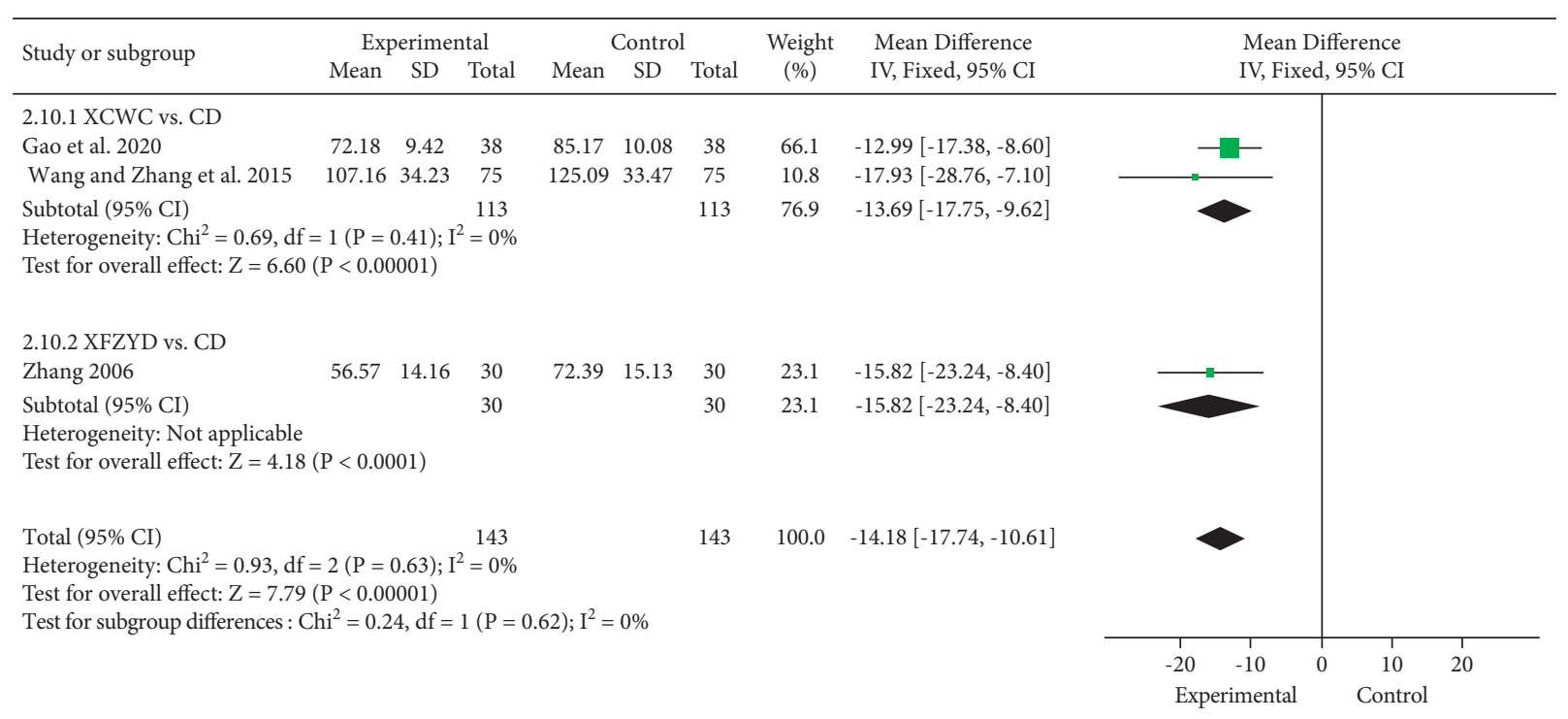

(b)

FIgURE 10: Forest plot of the trials showing vascular endothelial function indicators in different interventions: (a) NO; (b) ET.

effect of inhibiting platelet aggregation [52]. Chaihu had antioxidant, anti-inflammatory, and lipid-lowering effects, and it could regulate the blood coagulation state, which had a good effect on the treatment of cardiovascular diseases [53]. Gancao could significantly regulate lipid metabolism and play the purpose of treating atherosclerosis through antioxidation and anti-inflammatory effects [54].

XFZYD not only had anti-inflammatory, antioxidant, antiplatelet aggregation, and antitumor effects [55-57], but also could promote blood circulation, eliminate blood stasis, and make myocardial cells resistant to ischemic damage [58]. Furthermore, it could also improve microcirculation and vascular endothelial function and delay the formation of atherosclerosis [59]. Animal experiments found that XFZYD could not only reduce WBV and PV to improve hemorheology disorders, but also decrease TC, TG, and LDL-C and improve HDL-C [60]. And it could also prevent myocardial cells apoptosis probably by increasing the mRNA and protein expressions of SIRT1 and inhibiting the mRNA and protein expressions of P53, NF- $\kappa \mathrm{B}$, FoxO1, FoxO3, and FoxO4 [61]; in addition, it could promote angiogenesis and inhibit vascular remodeling moderately, the mechanism of which involved multiple pathways [62]. These research results also provided important data for demonstrating the potential mechanism of XFZYD for treatment of CHD.

Potential limitations of the included trials were related to the inconsistency and variability across eligibility criteria in the original trials, as well as the variability in study design, study type, sample size, and the inconsistency in methods of measurement used across trials. For example, most of the trials were conducted in China, and some of them did not include placebo control. In order to avoid the impact on the results, this study adopts more stringent methods of literature screening and data extraction. Although it may not 


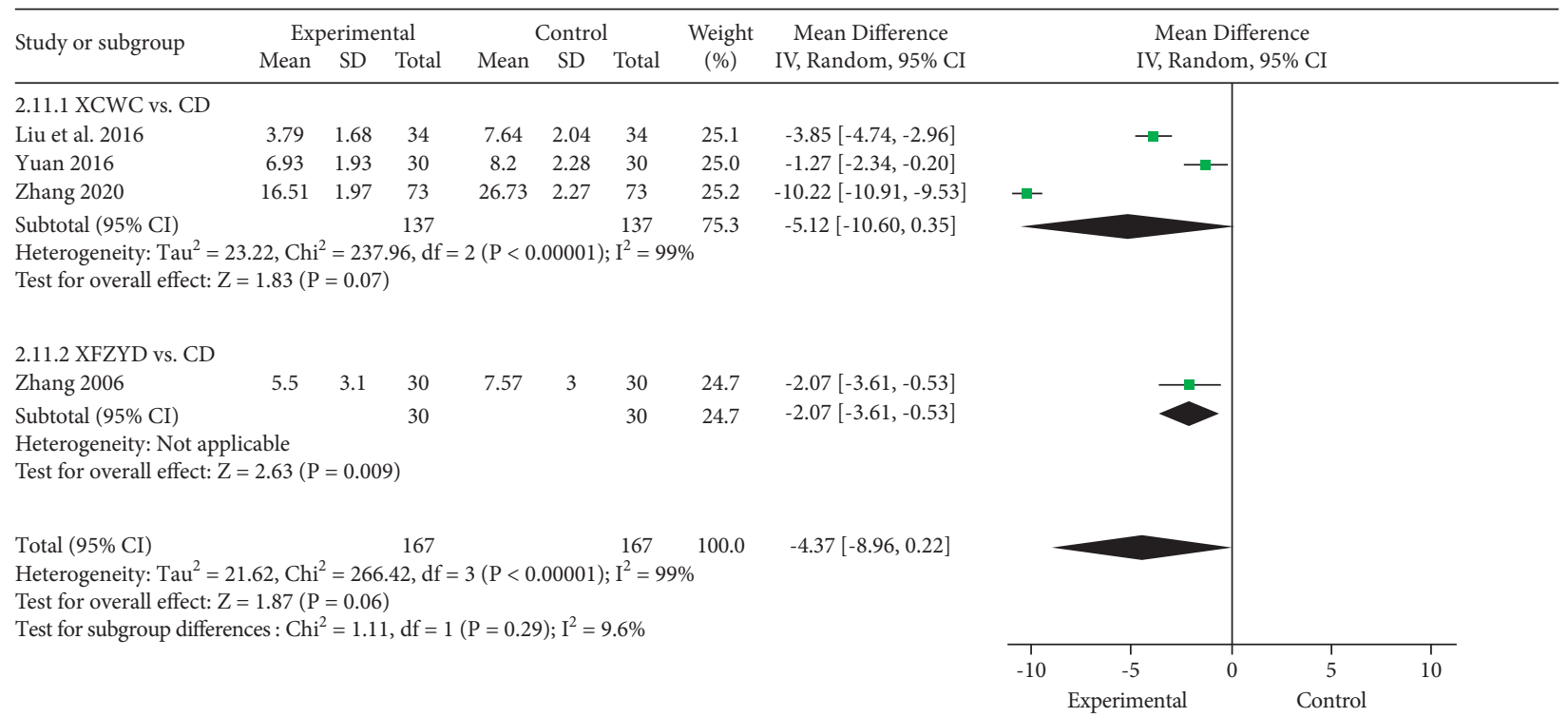

FIgURE 11: Forest plot of the trials showing TCM syndrome score in different interventions.

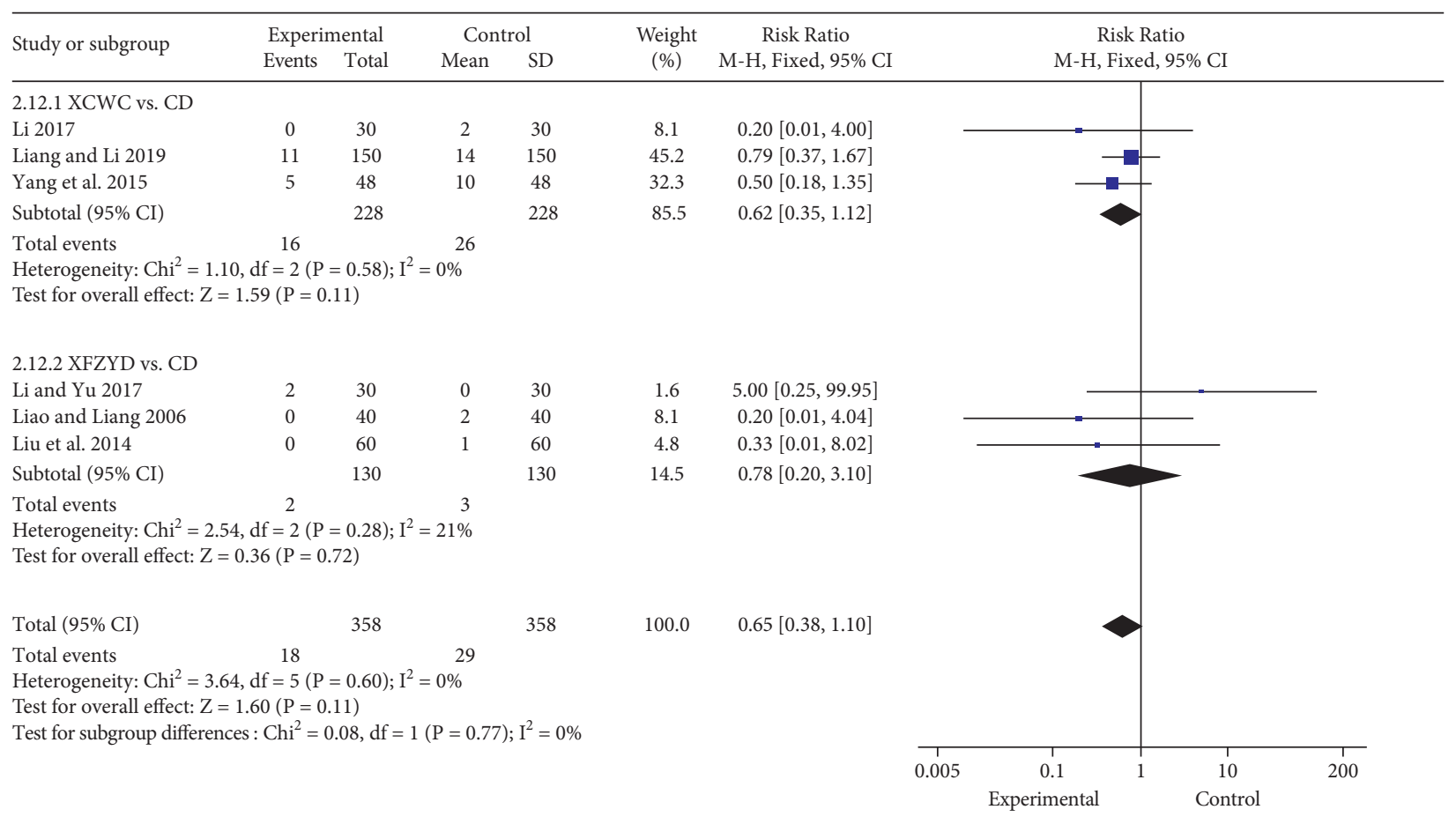

FIGURE 12: Forest plot of the trials showing adverse reaction in different interventions.

completely avoid the impact of risk, the effect trend of the research results can be used as a strong reference evidence.

\section{Conclusion}

In summary, multiple outcomes were used to systematically evaluate the efficacy and safety of XFZYD for CHD in this study. XFZYD could treat CHD through a comprehensive action of many herbs, which showed excellent efficacy with no obvious adverse reactions. And according to the results, $\mathrm{XFZYD}$ is more suitable for $\mathrm{CHD}$ patients with clinical indicators of dyslipidemia, high blood viscosity, or vascular endothelial dysfunction. Of course, more long-term, randomized, double-blind and multicenter clinical trials are anticipated to provide stronger evidence for XFZYD in the treatment of CHD in future.
Abbreviations
AF: $\quad$ Angina frequency
CD: Chemical drugs
CHD: Coronary heart disease 
CI: Confidence interval

DAP: Duration of angina pectoris

ET: Endothelin

FB: $\quad$ Fibrinogen

HDL-C: High-density lipoprotein cholesterol

LDL-C: Low-density lipoprotein cholesterol

MD: $\quad$ Mean difference

PV: $\quad$ Plasma viscosity

RR: $\quad$ Relative risk

TC: Total cholesterol

TCM: Traditional Chinese medicine

TG: $\quad$ Triglyceride

WBV: Whole blood viscosity

XCWC: $X u e-F u-Z h u-Y u$ decoction combined with chemical drug

XFZYD: Xue-Fu-Zhu-Yu decoction.

\section{Data Availability}

The data used to support the findings of this study are available from the corresponding author upon request.

\section{Conflicts of Interest}

The authors declare that they have no conflicts of interest.

\section{Authors' Contributions}

$\mathrm{SZ}$ and KWY were responsible for the conception and design of the study; SZ, ZLC, and YPT conducted the statistical analysis, drew the tables and pictures, and drafted the manuscript; SZ, ZLC, JLD, and KWY retrieved the database, screened the trials, extracted the data, and evaluated the methodological quality; and all authors critically revised the manuscript and approved the final version.

\section{Acknowledgments}

This study was funded by grants from General Program of National Natural Science Foundation of China (81873173 and 81974584), National Key Research and Development Project (2019YFC1708703), and Key Research and Development Program of Shaanxi (2019ZDLSF04-05).

\section{References}

[1] H. Mayu, S. Satoko, H. Takanori et al., "Small dense lowdensity lipoprotein cholesterol and the risk of coronary heart disease in a Japanese community," Journal of Atherosclerosis and Thrombosis, vol. 27, no. 7, pp. 669-682, 2020.

[2] G. M. Khandaker, V. Zuber, J. M. B. Rees et al., "Shared mechanisms between coronary heart disease and depression: findings from a large UK general population-based cohort," Molecular Psychiatry, vol. 25, no. 7, pp. 1477-1486, 2020.

[3] G. Chen, H. Q. He, K. Hu et al., "Sensitive biomarker analysis of Xue-Fu-Zhu-Yu capsule for patients with qi stagnation and blood stasis pattern: a nested case-control study," EvidenceBased Complementary and Alternative Medicine, vol. 2019, Article ID 7182865, , 2019.

[4] J. J. Zhao, H. H. Liu, B. Y. Xu et al., “The role of Xuefu Zhuyu decoction in prevention of contrast-induced nephropathy after percutaneous coronary intervention," Evidence-Based Complementary and Alternative Medicine, vol. 2020, Article ID 5419016, , 2020.

[5] D. Wang, P. Wang, R. Zhang et al., "Efficacy and safety of Xuefu Zhuyu decoction combined with Western medicine for angina pectoris in coronary heart disease: a protocol for systematic review and meta-analysis," Medicine (Baltimore), vol. 99, no. 50, Article ID e23195, 2020.

[6] T. Yang, X. Li, Z. Lu et al., "Effectiveness and safety of Xuefu Zhuyu decoction for treating coronary heart disease angina: a systematic review and meta-analysis," Medicine, vol. 98, no. 9, Article ID e14708, 2019.

[7] X. Yang, X. Xiong, G. Yang, and J. Wang, "Chinese patent medicine Xuefu Zhuyu capsule for the treatment of unstable angina pectoris: a systematic review of randomized controlled trials," Complementary Therapies in Medicine, vol. 22, no. 2, pp. 391-399, 2014.

[8] D. Moher, A. Liberati, J. Tetzlaff et al., "Preferred reporting items for systematic reviews and meta-analyses: the PRISMA statement," PLoS Medicine, vol. 6, no. 7, Article ID e1000097, 2009.

[9] J. P. T. Higgins and S. Green, Cochrane Reviewers' Handbook 5.3.0, Cochrane, London, UK, 2014, http://www. cochranehandbook.org.

[10] P. Sedgwick and L. Marston, "How to read a funnel plot in a meta-analysis," BMJ, vol. 16, Article ID h4718, 2015.

[11] B. Gao, R. An, and Y. Sun, "Evaluation of the effects on inflammatory factors, angiogenesis and endothelial function in patients with CHD by using Xuefu Zhuyu capsule," World Journal of Traditional Chinese Medicine, vol. 15, no. 7, pp. 1055-1058, 2020.

[12] J. Zhang, "The curative effect of Xuefu Zhuyu decoction combined with western medicine on angina pectoris of coronary heart disease and its effect on hemorheology," China Foreign Medical Research, vol. 18, no. 1, pp. 20-22, 2020.

[13] H. Huang, "Clinical observation on 20 cases of hyperlipidemia in coronary heart disease with qi stagnation and blood stasis type treated by Xuefu Zhuyu Jiaonang combined with routine western treatment," Journal of Gansu University of Chinese Medicine, vol. 36, no. 2, pp. 40-43, 2019.

[14] W. W. Kong and Y. Y. Liu, "Clinical observation of Xuefu Zhuyu decoction combined with western medicine in treating coronary heart disease with atrial fibrillation and blood stasis syndrome," Guangxi Journal of Traditional Chinese Medicine, vol. 42, no. 3, pp. 18-20, 2019.

[15] K. Liang and Q. F. Li, "Effect of decoction for removing blood stasis in the chest on clinical symptoms and platelet activating factor level in patients with coronary heart disease and angina pectoris," Chinese Journal of Public Health, vol. 18, no. 4, pp. 525-527, 2019.

[16] B. Xi and X. I. Li, "Clinical observation of Xuefu Zhuyu decoction combined with metoprolol sustained-release tablets in treating senile coronary heart disease and angina," Electronic Journal of Clinical Medicine Lit, vol. 6, no. 94, p. 48, 2019.

[17] H. X. Zhang, F. Z. Hou, and Q. Zhang, "Clincial study on Xuefu Zhuyu capsules combined with routine western medicine for angina pectoris of heart-bloostasis type," Journal of New Chinese Medicine, vol. 51, no. 10, pp. 90-93, 2019.

[18] N. An, "The evaluation on the clinical effect and analysis on the safety of Xuefu Zhuyu decoction in the treatment of stable angina pectoris," Chinese Medicine Modern Distance Education of China, vol. 16, no. 15, pp. 104-106, 2018. 
[19] Q. C. Zheng, “The effect of Xuefu Zhuyu decoction combined with atorvastatin on patients with stable angina pectoris combined with hyperlipidemia and blood lipids and hemorheology," Strait Pharmaceutical Journal, vol. 30, no. 8, pp. 210-212, 2018.

[20] G. S. Li, "Effect of Xuefu Zhuyu decoction on adhesion molecules in patients with unstable angina pectoris," Journal of Traditional Chinese Medical Sciences, vol. 36, no. 19, pp. 4-5, 2017.

[21] L. Li and S. Q. Yu, "Clinical observation of Suefu Zhuyu decoction in treating 30 cases of angina pectoris and blood stasis syndrome," Hunan Journal of Traditional Chinese Medical Sciences, vol. 33, no. 3, pp. 47-48, 2017.

[22] T. Y. Yang and J. Zhang, "Discussion on the curative effect of Xuefu Zhuyu Decoction on coronary heart disease unstable angina pectoris," World Latest Medicine Information, vol. 17, no. 57, pp. 168-173, 2017.

[23] J. H. Dong, "Observation on the influence of integrated traditional Chinese and western medicine on LDL-C and TC in treatment of coronary heart disease," Diet Health, vol. 3, no. 15, p. 92, 2016.

[24] D. F. Liu, M. L. Song, and S. J. Cheng, "Clinical observation on xuefuzhuyu decoction combined with western medicine in the treatment of angina pectoris of coronary heart disease," Asia-Pacific Traditional Medicine, vol. 12, no. 22, pp. 148-150, 2016.

[25] J. Q. Yuan, Study of Xuefuzhuyu Decoction on Treating Left Ventricular Diastolic Dysfunction of Coronary Heart Disease, pp. 1-37, Guangzhou University of Chinese Medicine, Guangzhou, China, 2016.

[26] J.-S. Zhang, Y.-Z. Wang, Y.-Q. Hu et al., "Effect of EphB4/ EphrinB2 reverse signal on angiogenesis induced by xuefu zhuyu capsule containing serum in human microvascular endothelial cell 1," Chinese Journal of Integrative Medicine, vol. 22, no. 8, pp. 605-610, 2016.

[27] C. H. Li, "Impact of xuefuzhuyu tang on angina of patients with coronary heart disease after interventional treatment," Chinese Journal of Integrated Traditional and Western Medicine, vol. 2, no. 16, pp. 91-92, 2015.

[28] L. Wang, "Application of Xuefu Zhuyu capsule in the treatment of patients with coronary heart disease," Chinese Journal of Modern Drug Application, vol. 9, no. 11, pp. 172-173, 2015.

[29] H. S. Wang, D. Y. Meng, and Y. J. Ge, "Thirty cases of unstable angina due to coronary atherosclerotic heart disease treated with Xuefu Zhuyu decoction," Henan Traditional Chinese Medicine, vol. 35, no. 8, pp. 1805-1806, 2015.

[30] K. Wang, J. Zhang, Y. D. Du et al., "Clinical observation of Xuefu Zhuyu capsule in treating elderly patients with acute myocardial infarction and restenosis after PCI," Practical Clinical Journal of Integrated Traditional Chinese and Western Medicine, vol. 15, no. 2, pp. 3-5, 2015.

[31] S. Q. Yang, X. M. Lv, J. H. Yang et al., "Clinical observation of Xuefu Zhuyu decoction in treating angina pectoris of coronary heart disease," Pharmacology and Clinical Chinese Material Medicines, vol. 31, no. 6, pp. 144-146, 2015.

[32] X. M. Chen, L. Ma, S. J. Cao et al., "Clinical observation of Xuefu Zhuyu capsule on patients with coronary heart disease and angina pectoris in August 2013," Medical Recapitulate, vol. 20, no. 2, pp. 331-333, 2014.

[33] X. Liu, Y. T. Li, D. J. Gu et al., "Influence Observation of Xue $\mathrm{Fu} \mathrm{Zhu} \mathrm{Yu}$ decoction on coronary heart disease angina pectoris patients blood fat," Chinese Medical Journal, vol. 29, no. 5, pp. 738-739, 2014.
[34] J. Wang, "Impact of xuefuzhuyu tang on angina of patients with coronary heart disease after interventional treatment," Cardiovascular Disease Electronic Journal of Integrated Traditional Chinese and Western Medicine, vol. 2, no. 16, pp. 91-92, 2014.

[35] C. L. Zhang and Y. X. Wang, "Twenty cases of stable angina pectoris of qi stagnation and blood stasis treated with xuefu zhuyu decoction and western medicine," Journal of Traditional Chinese Medical Researches, vol. 27, no. 7, pp. 20-21, 2014.

[36] S. J. Zhang, "Comparative study of the efficacy of different formulations of traditional Chinese medicine in treatment of blood stasis type of unstable angina," China Modern Medical and Clinical, vol. 19, no. 18, pp. 11-13, 2012.

[37] A. J. Li, "Clinical observation on 45 cases of coronary heart disease with blood stasis syndrome treated by xuefu zhuyu decoction," China Journal of Traditional Chinese Medicine and Pharmacy, vol. 17, no. 7, pp. 40-41, 2012.

[38] Z. H. Liao and Y. Liang, "Clinical observation of xuefu zhuyu oral liquid in treating angina pectoris of coronary heart disease," China Journal of Traditional Chinese Medicine and Pharmacy, vol. 3, no. 5, pp. 17-18, 2006.

[39] S. W. Zhang, Clinical Study on the Treatment of Cardiac Fibrosis of Coronary Heart Disease with Blood Stasis Syndrome by Promoting Blood Circulation, pp. 1-37, Guangzhou University of Chinese Medine, Guangzhou, China, 2006.

[40] W. Wang, X. C. Ma, X. F. Wang et al., "Clinical observation on treatment of 30 cases of coronary heart disease with angina pectoris by refined xuefu capsule," Journal of Traditional Chinese Medicine, vol. 41, no. 8, pp. 471-473, 2000.

[41] N. Shi, X. Han, W. Yu, L. Wang, and A. Lu, "Adoption in China of clinical practice guidelines for hypertension using traditional Chinese medical approaches: a literature review based on clinical studies," Journal of Alternative and Complementary Medicine, vol. 19, no. 1, pp. 1-8, 2013.

[42] W. H. Park, S. S. Shin, Y. C. Le, and C. H. Kim, "The inhibitory effects of Silso-San-Gami on atherosclerosis in KHC rabbits," Thrombosis Research, vol. 113, no. 3-4, pp. 235-241, 2004.

[43] X. Bai, W. X. Wang, R. J. Fu et al., "Therapeutic potential of hydroxysafflor yellow A on cardio-cerebrovascular diseases," Frontiers in Pharmacology, vol. 11, Article ID 01265, 2020.

[44] L. Zhang, J.-R. Du, J. Wang et al., "Z-ligustilide extracted from Radix Angelica Sinensis decreased platelet aggregation induced by ADP ex vivo and arterio-venous shunt thrombosis in vivo in rats," Yakugaku Zasshi, vol. 129, no. 7, pp. 855-859, 2009.

[45] Y.-h. Zhang, Y. Zhang, J. Li, W.-X. Tong, and F.-Q. Xu, "Protective effects of Xiongshao capsule on anti-inflammatory function of high-density lipoprotein in an atherosclerosis rabbit model," Chinese Journal of Integrative Medicine, vol. 23, no. 5, pp. 357-361, 2015.

[46] Y. Wang, G. Guo, B.-R. Yang et al., "Synergistic effects of Chuanxiong-Chishao herb-pair on promoting angiogenesis at network pharmacological and pharmacodynamic levels," Chinese Journal of Integrative Medicine, vol. 23, no. 9, pp. 654-662, 2017.

[47] R. Zhang, J. Zhou, M. Li et al., "Ameliorating effect and potential mechanism of rehmannia glutinosa oligosaccharides on the impaired glucose metabolism in chronic stress rats fed with high-fat diet," Phytomedicine, vol. 21, no. 5, pp. 607-614, 2014.

[48] C. Huang, Y. Cui, L. Ji et al., "Catalpol decreases peroxynitrite formation and consequently exerts cardioprotective effects 
against ischemia/reperfusion insult," Pharmaceutical Biology, vol. 51, no. 4, pp. 463-473, 2013.

[49] H. Negishi, S. Sugahama, A. Kawakamia et al., "Effect of angelica acutiloba extract on blood flow regulation in strokeprone spontaneously hypertensive rats," Natural product communications, vol. 11, no. 10, pp. 1497-1498, 2016.

[50] Y. C. Lin, Y. C. Lin, W. W. Kuo et al., "Platycodin D reverses pathological cardiac hypertrophy and fibrosis in spontaneously hypertensive rats," American Journal of Chinese Medicine, vol. 46, no. 3, pp. 537-549, 2018.

[51] S.-Y. Kim, H.-J. Kim, M.-K. Lee et al., "Naringin timedependently lowers hepatic cholesterol biosynthesis and plasma cholesterol in rats fed high-fat and high-cholesterol diet," Journal of Medicinal Food, vol. 9, no. 4, pp. 582-586, 2006.

[52] Y.-R. Jin, X.-H. Han, Y.-H. Zhang et al., “Antiplatelet activity of hesperetin, a bioflavonoid, is mainly mediated by inhibition of PLC- $\gamma 2$ phosphorylation and cyclooxygenase- 1 activity," Atherosclerosis, vol. 194, no. 1, pp. 144-152, 2007.

[53] H. Jiang, L. Yang, A. Hou et al., "Botany, traditional uses, phytochemistry, analytical methods, processing, pharmacology and pharmacokinetics of Bupleuri radix: a systematic review," Biomedicine and Pharmacotherapy, vol. 131, Article ID 110679, 2020.

[54] K. H. Nwe, A. Hamid, P. B. Morat et al., "Differential regulation of theoxidative 11 beta-hydroxystcroid dchydrogcnasc actively in tcstis and liver," Steroids, vol. 65 , no. 1, pp. 40-45, 2000.

[55] F. Meng, H. Lai, Z. Luo et al., "Effect of Xuefu Zhuyu decoction pretreatment on myocardium in sepsis rats," Evidence-Based Complementary and Alternative Medicine, vol. 2939307, 2008.

[56] X. Shi, H. Zhu, Y. Zhang, M. Zhou, D. Tang, and H. Zhang, "Xuefuzhuyu decoction protected cardiomyocytes against hypoxia/reoxygenation injury by inhibiting autophagy," BMC Complementary and Alternative Medicine, vol. 17, no. 1, p. 325, 2017.

[57] J. Liu, J. Zhang, L. Huan, X. Zhu, P. Hu, and W. Chen, "XuefuZhuyu tang exerts antitumor effects by inhibiting glioma cell metastasis and invasion via regulating tumor microenvironment," OncoTargets and Therapy, vol. 9, pp. 3603-3612, 2016.

[58] M. C. Zhang, "Clinical study of Xuefu Zhuyu decoction on unstable angina pectoris of coronary heart disease," Shanxi Journal of Traditional Chinese Medicine, vol. 32, no. 10, pp. 36-37, 2016.

[59] W. H. Xu, Y. Zhao, G. L. Ning et al., "Analysis of the molecular mechanism of Xuefu Zhuyu decoction in the treatment of coronary heart disease based on bibliometrics and systematic pharmacology," Chinese Traditional Patent Medicine, vol. 42, no. 11, pp. 3040-3046, 2020.

[60] X. B. Dou, T. Q. Han, Y. F. Zhao et al., "Effects of Xuefuzhuyu decoction on blood fat and myocardial enzymes in the coronary heart disease rat model," Guangdong Medical Journal, vol. 40, no. 6, pp. 767-771, 2016.

[61] M.-Q. Chen, K.-W. Yao, Z.-J. Liu, X.-X. Feng, and Y. Xiao, "Xuefu Zhuyu oral liquid prevents apoptosis of ischemic myocardium cells in rats by regulating SIRT1 and its pathwayrelated genes," Chinese Journal of Integrative Medicine, vol. 26, no. 6, pp. 442-447, 2020.

[62] W.-L. Shi, P.-F. Lu, D. Gao, J. Song, and K.-J. Chen, "Effect of Xuefu Zhuyu capsule on angiogenesis in hindlimb ischemic rats," Chinese Journal of Integrative Medicine, vol. 26, no. 1, pp. 39-45, 2020. 